\title{
Pac-Man bacteria eat nanoprobes for aggregation- enhanced imaging and killing diverse microorganisms
}

\section{Yunmin Yang}

Soochow university

\section{Binbin Chu}

Soochow university

\section{Jiayi Cheng}

Soochow university

\section{Jiali Tang}

Soochow university

\section{Bin Song}

Laboratory of Nanoscale Biochemical Analysis, Institute of Functional Nano and Soft Materials (FUNSOM), and Collaborative Innovation Center of Suzhou Nano Science and Technology, Soochow University

\section{Houyu Wang}

Soochow university

Yao He ( $\nabla$ yaohe@suda.edu.cn )

Soochow University

\section{Article}

Keywords: Pac-Man bacteria, microorganisms, microbial ecosystem

Posted Date: April 9th, 2021

DOI: https://doi.org/10.21203/rs.3.rs-272302/v1

License: (c) (i) This work is licensed under a Creative Commons Attribution 4.0 International License. Read Full License

Version of Record: A version of this preprint was published at Nature Communications on March 10th, 2022. See the published version at https://doi.org/10.1038/s41467-022-28920-6. 


\section{Abstract}

Currently optical-based techniques for in vivo microbial population imaging are limited by low imaging depth and highly light-scattering tissue; and moreover, are generally effective against only one specific group of bacteria. Here, we introduce an innovative Pac-Man imaging and therapy strategy, in which different bacteria displayed like Pac-Man actively eat the glucose polymer (GP)-modified gold nanoparticles through ATP-binding cassette $(A B C)$ transporter pathway, followed by laser irradiationmediated aggregation in the bacterial cells. As a result, the aggregates display 15.2-fold enhancement in photoacoustic signals and $~ 3.0$-fold enhancement in antibacterial rate compared with non-aggregated counterparts. Significantly, the developed Pac-Man strategy allows ultrasensitive imaging of as low as $10^{5}$ colony-forming unit (CFU) of bacteria in vivo, which is around two orders of magnitude lower than most optical contrast agents. We further demonstrate Pac-Man strategy enables the detection of $\sim 10^{7}$ CFU bacteria residing within tumour or gut. This technique enables visualization and treatment of diverse bacteria, setting the crucial step forward the study of microbial ecosystem.

\section{Introduction}

The mammalian microbiomes including Gram-positive and Gram-negative bacteria have lived in human bodies for millions of years and they have evolved with humans together. They can perceive changes of internal or external scenarios of human bodies, thus reflecting the level of human health and even causing several diseases ${ }^{1-3}$. Setting gut microbiomes as the example, they are associated with multiple human diseases, such as obesity, asthma, intestinal inflammation, multiple sclerosis, Parkinson's disease, cancer and so forth ${ }^{4-7}$. Furthermore, they can even determine whether cancer immunotherapy is effective ${ }^{8-9}$. Therefore, researchers have leveraged engineered bacteria to diagnose or treat diseases including cancer therapy ${ }^{10-13}$. To deeply understand the microbial communities as well as accurately control bacteria-based therapeutic or diagnostic methods, precisely imaging of their location within the host organism is a key determinant. However, existing approaches for in vivo bacterial location imaging are primarily based on optical reporter genes, organic dyes or nanoprobes, and in the case of deep tissue, their imaging performances would be greatly attenuated by low imaging depth and highly light-scattering tissue $^{14-17}$. In addition, most imaging agents are effective against only one specific group of bacteria, i.e., Gram-negative or Gram-positive bacteria, inevitably losing information of another group of bacteria $^{18-21}$.

Photoacoustic imaging (PAI) depends on PA effect, i.e., the thermal expansion of optical absorptive objects to generate ultrasound signals, overcoming the high scatter of optical photons in biological tissue ${ }^{22-23}$. Among a myriad of PAl agents, gold nanoparticles (AuNPs) have been extensively employed in PAI of mammalian cells due to their strong near-infrared (NIR) absorption, which can achieve deep penetration and distinct PA effect ${ }^{24-27}$. Of note, only these AuNPs larger than $50 \mathrm{~nm}$ feature near-infrared (NIR) absorption ${ }^{28-29}$. Paradoxically, nanoparticles with large size are not suitable for bacterial cell imaging since the size of bacterial cells is only 0.5-5 $\mu \mathrm{m}$, around one-tenth the size of mammalian cell. 
More seriously, distinguished from flexible outmost layer of mammalian cells, that is cellular membrane made of lipid bilayer, outmost layer of bacteria is relatively rigid envelope mainly composed of peptidoglycan. As a result, large-size AuNPs can passively enter mammalian cells via endocytosis, while they cannot freely access the bacterial intracellular volumes due to the formidable barrier of bacterial envelope ${ }^{30-32}$. It would be revolutionary if small-size AuNPs could robustly enter bacterial cells, followed by aggregation in intracellular volumes, thus exhibiting distinct photoacoustic signals for imaging of microbiome.

To address this contradiction, we present a novel Pac-Man imaging and therapy strategy for aggregationenhanced imaging and killing microorganisms in vivo. In such newly developed strategy, bacteria including Gram-negative as well as Gram-positive bacteria displayed like Pac-Man actively swallow their counterfeiting "foods", i.e., glucose polymer (GP)-conjugated gold nanoparticles (AuNPs) through bacteria-specific ABC transporter pathway (Fig. 1a). GP (e.g, poly[4-O-(a-D-glucopyranosyl)-Dglucopyranose) as the major "foods" (carbon source) for bacteria can be robustly internalized into bacterial cells through $A B C$ transporter ${ }^{33-37}$. As revealed in Fig. 1 b, $A B C$ transporter in E. coli consists of five subunits, i.e., LamB, MalE, MalF, MalG and MalK. Specifically, LamB is a typical outer membrane diffusion porin, MalE is the major recognition site for linearly $a(1-4)$-glucosidically linked GP (e.g., amylose, maltotriose, cyclodextrins, etc.), MalF and MalG are two tightly membrane-bound permease subunits, and MalK is the ATP-hydrolyzing subunit of the transporter ${ }^{38-47}$. As confirmed by transmission electronic microscopy (TEM) images, numerous dispersed nanoparticles distribute in both M. luteus (ML) and E. coli (EC) intracellular volumes (Second column in Fig. 1c) when M. luteus and E. coli are respectively incubated with GP-linked nanoparticles at $37^{\circ} \mathrm{C}$ for $2 \mathrm{~h}$, and then washed with PBS buffer. On the contrary, no nanoparticles appear in bacterial cells if the surface of nanoparticles is not modified with GP molecules (First column in Fig. 1c). The TEM results directly demonstrate that the nanoprobes could be internalized into bacterial cells rather than nonspecifically absorbed on the cell surface.

Under the laser irradiation, the internalized AuNPs further aggregate into larger ones when their surfaces are modified with photo-reactive amino acid analogs (e.g., NHS-diazirine) ${ }^{28,48-50}$. Also confirmed by TEM images, aggregated nanoparticles appear in the bacterial intracellular volumes after the laser irradiation (Third column in Fig. 1c). By further leveraging drug loading abilities of AuNPs (e.g., chlorin e6 (Ce6)), such Pac-Man strategy allows not only dual-modal imaging, i.e., fluorescence imaging $(\mathrm{FI})$ and photoacoustic imaging (PAl), but also combination therapy, i.e., photothermal therapy (PTT) and photodynamic therapy (PDT) against bacteria (Fig. 1a \& 1b). In the case of imaging and therapeutic performance, the efficacy for the aggregates significantly enhances compared with non-aggregated counterparts (e.g., 15.2-fold enhancement in photoacoustic signals, 3.0-fold enhancement in antibacterial rates). We further demonstrate the developed Pac-Man strategy facilitate imaging of bacteria in proof-of-concept models of tumour xenografts and gastrointestinal tract. As such, our work offers a simple and general strategy for probing the in vivo location of microbial populations as well as treating them. 


\section{Results}

Synthesis and characterization of nanoprobes. The as-synthesized nanoprobes are composed of four modules, those are AuNPs, GP, diazirine and Ce6. As schematically illustrated in Fig. 2a, we firstly prepare GP-conjugated AuNPs (GP-AuNPs) through the Schiff base reaction, in which the aldehyde groups of GP $\left(20 \mathrm{mg} \mathrm{mL}^{-1}, 100 \mu \mathrm{L}\right)$ react with amino groups on AuNPs $\left(1.5 \mathrm{mg} \mathrm{mL}^{-1}, 200 \mu \mathrm{L}\right)$ surface to form Schiff base and then reduced by $\mathrm{NaBH}_{4}$ to form stable structure ${ }^{51}$. Next, we obtain Ce6-loaded GP-AuNPs (GPAuNPs@Ce6) through electrostatic adsorption between Ce6 $\left(1.0 \mathrm{mg} \mathrm{mL}^{-1}, 10 \mu \mathrm{L}\right)$ and GP-AuNPs (1.0 mg $\mathrm{mL}^{-1}, 300 \mu \mathrm{L}$ ). According to the characterizations of TEM, dynamic light scattering (DLS), Zeta potential and ultraviolet (UV) (Supplementary Fig. 1),we successfully synthesize GP-AuNPs@Ce6. Finally, we modify the surfaces of GP-AuNPs@Ce6 with NHS-diazirine molecules to attain the nanoprobes (GPdAuNPs@Ce6) through the established condensation reaction ${ }^{28}$. Upon the irradiation of $405 \mathrm{~nm}$-laser, the modified diazirine are transformed into carbene moieties, which are easy to form covalent bonds among each other, leading to the aggregated products $28,57,58$. Figure $2 \mathrm{~b}$ shows irradiation time-dependent TEM images of GP-dAuNPs@Ce6 (e.g., 405 nm, 1.0 W cm-2). At the beginning of irradiation, we can observe spherical nanoparticles ( $11 \mathrm{~nm}$ in diameter) with good dispersibility. With the increase of irradiation time, the nanoparticles gradually aggregate with each other. When the irradiation time arrives at $25 \mathrm{~min}$, the aggregated nanoparticles feature a much larger diameter of $\sim 150 \mathrm{~nm}$. Consistent with TEM results, DLS results show that the hydrodynamic size increases from $\sim 12 \mathrm{~nm}$ to $\sim 160 \mathrm{~nm}$ after 25 -min irradiation (Fig. 2c). Also, the surface plasmon resonance (SPR) peak of nanoparticles initially locates at $\sim 520 \mathrm{~nm}$ while gradually shifts to a longer wavelength during irradiation (Fig. 2d). Of note, the narrow peak of SPR gradually becomes as a broad shoulder after 10-min irradiation, and the maximum absorption peak appears at $~ 700 \mathrm{~nm}$ after 25 -min irradiation, also suggesting the formation of aggregated plasmonic nanoparticles ${ }^{28}$. Accordingly, the color of the nanoprobe solution changes from wine red (Fig. 2d-I) to bluish gray (Fig. 2d-II). In contrast, there are no obvious changes in TEM images, DLS analysis and absorption spectra of nanoprobes without photoreactive crosslinkers before and after laser irradiation (Supplementary Fig. 2). These experimental results demonstrate we can controllably aggregate the synthesized nanoprobes through photo-crosslinking reactions.

Due to the agglomeration-enhanced effects, the aggregated nanoprobes exhibit superior properties compared to their non-aggregated counterparts. As depicted in Fig. 2e, the aggregated GP-dAuNPs@Ce6 exhibits 4.1-fold enhancement in fluorescence intensity compared with GP-dAuNP@Ce6 under the identical conditions. As expected, the aggregated GP-dAuNPs@Ce6 features better photothermal therapy (PTT) effects compared with GP-dAuNP@Ce6. Typically, the temperature of aggregated GP-dAuNPs@Ce6 solution rises to $52^{\circ} \mathrm{C}$ under 808 -nm laser irradiation for $5 \mathrm{~min}$, while the temperature of GP-dAuNP@Ce6 solution only improves to $33^{\circ} \mathrm{C}$ under the same conditions (Fig. 2f). In addition, the aggregated GPdAuNPs@Ce6 displays distinct photodynamic therapy (PDT) ability, comparable to that of GPdAuNPs@Ce6 and free Ce6 molecules at the same Ce6 concentration under 660-nm irradiation (Fig. 2g). 
As we know, 405-nm light belongs to short-wavelength light and has weak penetrating ability. Notwithstanding, according to previous reports, about $40 \%$ of $405-\mathrm{nm}$ laser with $1.0 \mathrm{~W} \mathrm{~cm}^{-2}$ still penetrates the tissue when the thickness of the tissue is more than $3 \mathrm{~mm}$, which is enough to trigger the aggregation of nanoparicles ${ }^{27,28}$. In this context, we first study the tissue penetration depth of the constructed probes in vitro by detecting photoacoustic signals. As shown in Fig. $2 \mathrm{~h}$, the photoacoustic signals of GP-dAuNPs@Ce6 with 405-nm laser irradiation (GP-dAuNPs@Ce6 (+)) gradually become weak along with the increase of chicken breast tissue thickness from 0 to $5 \mathrm{~mm}$. When the thickness of chicken breast tissue even reaches to $4 \mathrm{~mm}$, the photoacoustic intensity of GP-dAuNPs@Ce6 (+) is still significantly stronger than other three groups $(p=0.0007)$ (Fig. 2i). Such tissue penetration depth provides a guarantee for the subsequent in vivo animal experiments. These unique merits of nanoprobes lay foundation for their applications in aggregation-enhanced imaging and treatments against bacteria.

Pac-Man bacteria eat nanoprobes. Next, we confirm Pac-Man bacteria indeed eat the as-synthesized nanoprobes of GP-dAuNPs@Ce6 (Fig. 3a). In addition to TEM characterizations (Fig. 1c), we perform confocal laser scanning microscope (CLSM) experiments when Gram-positive bacteria of $S$. aureus (SA), M. luteus (ML), and Gram-negative bacteria of E. coli (EC), P. aeruginosa (PA) are respectively incubated with 1.0 mg mL m $^{-1}$ of dAuNPs@Ce6, GP-AuNPs@Ce6 or GP-dAuNPs@Ce6 at $37^{\circ} \mathrm{C}$ for 2 h, and then washed with PBS buffer. As shown in CLSM images in Fig. 3b-3e, both green fluorescence signals originated from AuNPs (first column, $\lambda_{\mathrm{ex}}=405 \mathrm{~nm}, \lambda_{\mathrm{em}}=500-560 \mathrm{~nm}$ ) and red fluorescence signals originated from Ce6 (second column, $\lambda_{\mathrm{ex}}=405 \mathrm{~nm}, \lambda_{\mathrm{em}}=600-680 \mathrm{~nm}$ ) can be clearly observed in all GPAuNPs@Ce6 or GP-dAuNPs@Ce6-treated bacteria. Furthermore, green signals overlap well with red signals in the merged channel (third column), demonstrating the good co-localization between AuNPs and Ce6, and thus indicating the good stability of nanoprobes when they are incubated with bacteria. However, neither green nor red fluorescence signals are detected in dAuNPs@Ce6-treated bacteria due to the absence of GP molecules in nanoprobes for targeting bacteria (Supplementary Fig. 3). To further investigate whether Pac-Man bacteria eat nanoprobes via ABC transporter pathway, we perform inhibition assay as well as competition assay. In the inhibition assay, we can not detect the fluorescence signals of GP-dAuNPs@Ce6 in the bacteria when the bacteria are treated with the bacteria respiratory chain inhibitor (e.g., sodium azide $\left(\mathrm{NaN}_{3}\right)$ ) (Supplementary Fig. 4) ${ }^{52}$. In the competition assay, we observe that the fluorescence signals of GP-dAuNPs@Ce6 in bacteria gradually weaken when the bacteria are respectively incubated with GP with concentrations of 0,20 or $100 \mathrm{mg} \mathrm{mL}^{-1}$ for 5 min in advance (Supplementary Fig. 5). Both the results of inhibition assay and competition assay demonstrate the uptake mechanism of nanoprobes into bacteria is indeed through $A B C$ transporter pathway. To further verify the specificity of synthesized nanoprobes towards bacteria over mammalian cells, COS-7 and U87MG cells are incubated with $1.0 \mathrm{mg} \mathrm{mL}^{-1} \mathrm{GP}$-dAuNPs@Ce6 at $37^{\circ} \mathrm{C}$ for $2 \mathrm{~h}$, and then washed with PBS buffer. As expected, we can not observe fluorescence signals in treated COS-7 and U87MG cells (Supplementary Fig. 6), suggesting the nanoprobes are hardly internalized into mammalian cells during 2-h incubation.

It is worth pointing out that fluorescence signals of all kinds of bacteria treated with GP-dAuNPs@Ce6 after 405-nm laser irradiation for 25 min exponentially enhance (Fig. 3e) compared with those of GP- 
AuNPs@Ce6-treated groups without (Fig. 3b) or with laser irradiation (Fig. 3c),GP-dAuNPs@Ce6-treated groups without laser irradiation (Fig. 3d). It indicates that the GP-AuNPs@Ce6 might aggregate with the assistance of photoreactive crosslinkers. More quantitatively, the mean fluorescence intensity in GPdAuNPs@Ce6-treated S. aureus (SA) after laser irradiation is 5.2-fold stronger than that of other three groups $(p<0.0001)$ (Fig. $3 f-i)$, in accordance with above CLSM imaging analysis. Similar results can be observed in other three kinds of bacteria. These results demonstrate that the nanoprobes eaten by bacteria are ready for aggregation-enhanced fluorescent imaging of diverse bacteria.

Aggregation-enhanced imaging of bacteria in superficial tissues. Next, we first prove that the proposed Pac-Man strategy enables aggregation-enhanced imaging of diverse bacteria in surface skin tissue. After the 24-h injection of $50 \mu \mathrm{L} \mathrm{SA}$ or PA into right or left caudal thigh of the mice, the infected mice are intravenously injected with $100 \mu \mathrm{L}$ of $1.0 \mathrm{mg} \mathrm{mL}^{-1}$ GP-dAuNPs@Ce6 (Fig.4a) or GP-AuNPs@Ce6 (Supplementary Fig. 7a). The infected sites then would be irradiated by a laser ( $405 \mathrm{~nm}, 25 \mathrm{~min}$ ), which are imaged by an in vivo optical imaging system (IVIS Lumina III) $\left(\lambda_{\mathrm{ex}}=460 \mathrm{~nm}, \lambda_{\mathrm{em}}=670 \mathrm{~nm}\right.$ ) at 24-h postinjection. The SA or PA concentration at the infection site during imaging is $\sim 1.0 \times 10^{7} \mathrm{CFU}$, which is determined via tissue harvesting, homogenization, and culturing with CFU count ${ }^{53-54}$. As revealed in Fig. 4a, we can observe fluorescence signals at both two infected sites. Of note, the fluorescence intensity in groups treated by GP-dAuNPs@Ce6 upon laser irradiation substantially improves, 2.3 fold higher than that of groups without laser irradiation $(p<0.0001)$. On the contrary, there are no significant changes in fluorescence intensity of infected site treated by GP-AuNPs@Ce6 with or without laser irradiation ( $p$ > 0.05) (Supplementary Fig. 7a).

After 24-h injection of $50 \mu \mathrm{L}$ PBS buffers or bacteria mixture (PA + SA) into left or right caudal thigh of mice, the infected mice are intravenously injected with $100 \mu \mathrm{L}$ of $1.0 \mathrm{mg} \mathrm{mL}^{-1}$ GP-dAuNPs@Ce6 (Fig. 4b) or GP-AuNPs@Ce6 (Supplementary Fig. 7b) and then imaged at 24-h post-injection. The PA + SA concentration during imaging is $\sim 1.0 \times 10^{7} \mathrm{CFU}$. As revealed in Fig. 4b, we can only observe distinct fluorescence signals at the (PA $+S A)$-infected site rather than PBS-treated site. Consistently, the fluorescence intensity in groups treated by GP-dAuNPs@Ce6 upon laser irradiation is 2.2 fold higher than that of groups without laser irradiation $(p<0.0001)$. In contrast, there are no significant changes in fluorescence intensity of infected site treated by GP-AuNPs@Ce6 with or without laser irradiation ( $p>$ 0.05) (Supplementary Fig. 7b). Furthermore, no obvious fluorescence signals can be measured in the infected sites when they are treated with AuNPs or dAuNPs@Ce6 (Supplementary Fig. 8). Together, these results indicate the nanoprobes of GP-dAuNPs@Ce6 aggregate at the infected site after laser irradiation, greatly enhancing the fluorescence imaging performance.

To determine the detection limit of Pac-Man strategy, we image bacteria with a series of concentrations. Remarkably, we detect distinct fluorescent signals of SA (Fig. 4c) or PA (Fig. 4d) cells at concentrations as low as $~ 1.0 \times 10^{5} \mathrm{CFU}$ in vivo by using GP-dAuNPs@Ce6 after 405-nm laser irradiation, which is around two orders of magnitude lower than most contrast agents (e.g., nuclease-activated probes, zincdipicolylamine probes, supramolecular nanoassemblies and antimicrobial peptides, etc) ${ }^{59-61}$. Also, the 
fluorescence intensity of GP-dAuNPs@Ce6 groups with laser irradiation is 2.2 fold higher than that without laser irradiation $(p<0.0001)$. Hence, Pac-Man strategy features an ultrahigh sensitivity, which should be sufficient for many in vivo scenarios.

Next, we test the feasibility of Pac-Man strategy for photoacoustic imaging of diverse bacteria in surface tissues by using a photoacoustic imaging system (Vevo®LAZR, VisualSonics, Inc., Canada) at 24-h postinjection of GP-dAuNPs@Ce6. As shown in Fig. 4e, SA or PA cells at concentrations as low as $1.0 \times$ $10^{5}$ CFU generate negligible photoacoustic signals in AuNPs, dAuNPs@Ce6 and GP-AuNPs@Ce6-treated groups before and after laser irradiation, and in GP-dAuNPs@Ce6-treated groups before laser irradiation. In sharp contrast, SA or PA cells at concentrations as low as $\sim 1.0 \times 10^{5} \mathrm{CFU}$ generate much stronger photoacoustic signals in GP-dAuNPs@Ce6-treated groups after laser irradiation, and the signal intensity has 15.2-fold enhancement compared with other groups $(p<0.0001)$. These results suggest Pac-Man strategy feature excellent photoacoustic imaging ability for mapping bacteria in surface tissues.

Aggregation-enhanced imaging of bacteria in tumour and gut. Next, we verify the effectiveness of PacMan strategy on imaging of bacteria in tumour and gut. Accordingly, we construct two different kinds of proof-of-concept models of bacteria in tumour xenografts and gastrointestinal tract. To construct the tumour xenografts model, we subcutaneously inject $100 \mu \mathrm{L}$ of 4T1-LUC cells $\left(\sim 5 \times 10^{6}\right.$ cells) into the right back region of female nude mice (6-8 weeks old). When the tumour grows to $100 \mathrm{~mm}^{3}$, we subcutaneously inject $50 \mu \mathrm{L}$ of SA or PA into the left thigh region of mice (Fig. $5 \mathrm{a} \& 5 \mathrm{~b}$ ) or respectively into both the left thigh region and the right tumour region of mice (Fig. $5 \mathrm{c} \& 5 \mathrm{~d}$ ), followed by intravenous injection of $100 \mu \mathrm{L}$ GP-dAuNPs@Ce6 $\left(1.0 \mathrm{mg} \mathrm{mL}^{-1}\right)$. The infected sites as well as tumour sites are then imaged by an in vivo optical imaging system $\left(\lambda_{\mathrm{ex}}=460 \mathrm{~nm}, \lambda_{\mathrm{em}}=670 \mathrm{~nm}\right)$ or a photoacoustic imaging system at 24-h postinjection of GP-dAuNPs@Ce6. The bacterial cell concentration during imaging is 1.0 $\times 10^{7} \mathrm{CFU}$, which is within the range of certain commensal and therapeutic scenarios ${ }^{1,62,63}$. As revealed in Fig. 5a \& 5b, we can observe fluorescence and photoacoustic signals only at the infected sites instead of tumour sites containing no bacteria, indicating the Pac-Man strategy enables the discrimination of bacteria from tumour. Expectedly, the detecting signals from the infected sites treated with 405-nm laser irradiation are significantly stronger than counterparts without 405-nm laser irradiation (e.g., 1.5 (SA) and $~ 2.8$ (PA)-fold enhancement in fluorescence signals, 2.6 (SA) and 4.0 (PA)-fold enhancement in photoacoustic signals) $(p<0.0001)$. As further revealed in Fig. $5 c \& 5 d$, we can observe fluorescence and photoacoustic signals simultaneously at the infected sites and the tumour sites containing bacteria. Consistently, the detecting signals from both the infected sites and the tumour sites containing bacteria treated with 405-nm laser irradiation are much stronger than counterparts without 405-nm laser irradiation (e.g., 3.19 (SA, Left), 4.66 (SA, Right) and 2.4 (PA, Left), 2.96 (PA, Right)-fold enhancement in fluorescence signals, 3.08 (SA, Left), 2.67 (SA, Right) and 3.3 (PA, Left), 4.5 (PA, Right)-fold enhancement in photoacoustic signals) $(p<0.0001)$. These results together prove the PacMan strategy allows the aggregation-enhanced imaging of diverse bacteria residing within tumour tissues. 
To construct gastrointestinal tract model, the agarose gel containing E.coli (EC) is injected into the gut lumen of the female nude mice (6-8 weeks old). Afterwards, $100 \mu \mathrm{L}$ of $1.0 \mathrm{mg} \mathrm{mL}^{-1}$ GP-dAuNPs@Ce6 is intravenously injected into the mice. At 24-h postinjection of GP-dAuNPs@Ce6, the gut is then imaged by an in vivo optical imaging system $\left(\lambda_{\mathrm{ex}}=460 \mathrm{~nm}, \lambda_{\mathrm{em}}=670 \mathrm{~nm}\right)$ or a photoacoustic imaging system. The final concentration of EC is $\sim 1.0 \times 10^{7} \mathrm{CFU}$ during imaging. Indeed, distinct fluorescence as well as photoacoustic signals are measured in the gut containing EC (Fig. 5e). Also, the fluorescence intensity from the gut containing bacteria treated with $405-\mathrm{nm}$ laser irradiation is $~ 1.6-$ fold stronger than that the counterparts without 405-nm laser irradiation. Consistently, the photoacoustic intensity from the gut containing bacteria treated with 405-nm laser irradiation is 5.5-fold higher than that the counterparts without 405-nm laser irradiation. These data demonstrate the Pac-Man strategy could resolve the spatial distribution of bacteria within the gut.

Aggregation-enhanced therapy of bacteria in vitro. Next, we evaluate the in vitro antibacterial activity of the developed Pac-Man strategy. As expected, wrinkled or lysed EC (Fig. 6a) and SA cells (Fig. 6b) exhibit in scanning electron microscope (SEM) images when they are incubated with GP-dAuNPs@Ce6 for $2 \mathrm{~h}$ and then suffered with a series of laser irradiations (i.e., $405 \mathrm{~nm}, 1.0 \mathrm{~W} \mathrm{~cm}^{-2}, 25 \mathrm{~min} ; 660 \mathrm{~nm}, 12 \mathrm{~mW}$ $\mathrm{cm}^{-2}, 5 \mathrm{~min} ; 808 \mathrm{~nm}, 1.0 \mathrm{~W} \mathrm{~cm}^{-2}, 5 \mathrm{~min}$ ), while the intact EC and SA cells exist in other control groups. As further revealed in agar plate experiments, small amount of bacterial colony of SA (Fig. 6c) or EC (Fig. 6d) exists in GP-dAuNPs@Ce6-treated groups after the order 405-nm (25 min), 660-nm (5min) and 808-nm (5 $\mathrm{min}$ ) laser irradiations. During these irradiation processes, $405-\mathrm{nm}$ laser leads to the aggregation of nanoprobes, 660-nm laser induces Ce6 to produce singlet oxygen (photodynamic therapy (PDT) effects) ${ }^{55-56}$, and 808-nm laser trigger aggregated AuNPs to yield thermal (photothermal therapy (PTT) effects ${ }^{28}$. By contrast, numerous bacterial colonies are observed in other control groups. As further quantitatively analyzed in Fig. 6e-6f, GP-dAuNPs@Ce6 shows dominant in vitro antibacterial rates of $94.5 \%$ to $S A, \sim 97.6 \%$ to EC. These results demonstrate that Pac-Man strategy possesses an aggregationenhanced anti-bacterial ability against both Gram-negative and Gram-positive bacteria in vitro.

To assess the antibacterial rates of the nanoprobes, we excise the infected tissues from the mice after the therapy, followed by homogenization, and culturing with CFU count.

Aggregation-enhanced therapy of bacteria in vivo. In order to evaluate the antibacterial ability of Pac-Man strategy in vivo, $50 \mu \mathrm{L}$ of SA and PA are injected into the right thigh of the mice, respectively. Then, these infected mice are intravenously injected with $100 \mu \mathrm{L}$ of GP-dAuNPs@Ce6 $(1.0 \mathrm{mg} / \mathrm{mL})$ respectively. The bacterial cell concentration is $\sim 1.0 \times 10^{7} \mathrm{CFU}$ during treatment. For systematic comparisons, these mice are then divided into six therapy groups (e.g., group 1 (G1): GP-dAuNPs@Ce6 +660-nm laser; group 2 (G2): GP-dAuNPs@Ce6 + 808-nm laser; group 3 (G3): GP-dAuNPs@Ce6 + 660-nm laser + 808-nm laser; group 4 (G4): GP-dAuNPs@Ce6 + 405-nm laser+660-nm laser; group 5 (G5): GP-dAuNPs@Ce6 + 405-nm laser + 808-nm laser; group 6 (G6): GP-dAuNPs@Ce6 + 405-nm laser + 660-nm laser + 808-nm laser. The representative photographs of these mice are displayed in Figs. 7a \& 7e. As expected, earliest and fastest wound healing and scarring occurs in $G 6$, which is further confirmed by the relative wound area $\left(S / S_{0}\right)$ in Figs. $7 \mathrm{~b} \& 7 \mathrm{f}$. To assess the antibacterial rates of the nanoprobes, we excise the infected tissues from the 
mice after the therapy, followed by homogenization, and culturing with CFU count. In line with therapy results, CFU counts in G6 are significantly less than those of other 5 groups $(p<0.0001)$ (Figs. 7c \& 7g). As a consequence, the in vivo antibacterial rates are calculated as $97.3 \%$ against $S A$ and $98.1 \%$ against PA. The high antibacterial rates are contributed to PDT as well as PTT effects. Afterwards, a series of staining experiments including hematoxylin-eosin staining, Masson's trichrome and Gram-related staining of infected tissues from the six groups after therapy are performed. As manifested in Figs. 7d \& 7h, compared with other groups, almost no cell necrosis (H\&E) and clear tissue texture, no inflammatory factors (Massion) and no obvious bacteria (Gram) are found in G6. On the other aspect, the PTT effects are directly confirmed by an IR thermal imaging camera. As revealed in Figs. 7i \& 7j, the local temperature of the infected tissues treated with GP-dAuNPs@Ce6 dramatically rises to $52^{\circ} \mathrm{C}$ after $808 \mathrm{~nm}$-irradiation. In contrast, the local temperature of the tumour hardly changes in other control groups under the identical treatments. These results together prove that Pac-Man strategy shows an aggregation-enhanced antibacterial ability against both Gram-negative and Gram-positive bacteria in vivo.

Toxicity assessment. Furthermore, we test the cytotoxicity and in vivo toxicity of GP-dAuNPs@Ce6. We examine the cytotoxicity of nanoprobes via the established methyl thiazolyl tetrazolium (MTT) assays. As revealed in Supplementary Fig. 9, the cell viability of normal cells (e.g., LO2, HEK-293T and Marc-145 cells) as well as cancer cells (e.g., HeLa and MCF-7 cells) is above $80 \%$ even when they are incubated with 2.0 mg mL ${ }^{-1}$ GP-dAuNPs@Ce6 for 24 h, suggesting the low-cytotoxicity of GP-dAuNPs@Ce6 in vitro. We examine the in vivo toxicity of nanoprobes via hematoxylin-eosin, Masson's trichrome and Gram staining. As presented in Supplementary Fig. 10, no hydropic degeneration occurs in the heart tissues; no inflammatory infiltrates appear in the liver tissues; no hyperplasia exists in the spleen tissues; no pulmonary fibrosis is found in the lung tissues; glomerula structures are easily identified in the kidney tissues. Together, no obvious histopathological abnormalities are found in biopsy sections in all resected organs, indicating the feeble in vivo toxicity of the GP-dAuNPs@Ce6.

\section{Conclusion}

In summary, we successfully construct a novel diagnosis and treatment strategy, in which Pac-Man bacteria eat nanoprobes for aggregation-enhanced imaging and killing diverse bacteria in vivo. The nanoprobes are made of GP, diazirine and Ce6 modified AuNPs. Thanks to the bacteria-specific ABC transporter pathway, the nanoprobes can be robustly and selectively internalized into both Gram-negative and Gram-positive bacterial cells, while hardly entering mammalian cells. Owing to the photo-active crosslinkers of diazirine, the internalized nanoprobes can aggregate with each other upon 405-nm laser irradiation, displaying dramatically enhanced fluorescence signals as well as photoacoustic signals. Consequently, as few as $\sim 1.0 \times 10^{5} \mathrm{CFU}$ of bacteria is able to be discriminated in vivo, which is around two orders of magnitude lower than most optical contrast agents. Using the Pac-Man strategy, we successfully detect diverse bacteria at cell concentrations of $\sim 1.0 \times 10^{7} \mathrm{CFU}$ residing within tumour or gut. Such high sensitivity is enough for many in vivo scenarios. Moreover, the Pac-Man strategy exhibits ultrahigh in vivo broad-spectrum antibacterial efficiency more than $\sim 95.0 \%$. Taken together with excellent 
biocompatibility, this kind of high-performance strategy holds high promise for the study of microbiome in tissues and the development of new diagnostic and therapeutic agents.

\section{Methods}

\section{Fabrication of GP-dAuNPs@Ce6.}

The amino-terminated gold nanoparticles ( $\mathrm{NH}_{2}$-AuNPs) (diameter: $10 \mathrm{~nm}$ ), $\mathrm{NHS}$-diazirine, $\mathrm{NaBH}_{4}$, chlorin e6 (Ce6) and glucose polymer (GP) (e.g., poly[4-O-(a-D-glucopyranosyl)-D-glucopyranose]) were purchased from Sigma-Aldrich (Shanghai, China). All chemicals were analytical grade and used without additional purification. The AuNPs solution $\left(1.5 \mathrm{mg} \mathrm{mL}^{-1}, 200 \mu \mathrm{L}\right)$ was mixed with the GP dissolved in deionized water $\left(20 \mathrm{mg} \mathrm{mL}^{-1}, 100 \mu \mathrm{L}\right)$. The dispersion was continuously stirred at $70{ }^{\circ} \mathrm{C}$ for $6 \mathrm{~h}$, and $0.02 \mathrm{mg}$ of $\mathrm{NaBH}_{4}$ was added and reacted for another $12 \mathrm{~h}$ at room temperature to obtain the stable GP-modified AuNPs (GP-AuNPs). In order to remove the unreacted GP molecules, the reaction solutions were further purified by simple centrifugation (15000 rpm, 20min) for over three times. To further fabricate the GPAuNPs@Ce6, the Ce6 solution ( $\left.1.0 \mathrm{mg} \mathrm{mL}^{-1}, 10 \mu \mathrm{L}\right)$ was added in the above GP-AuNPs solution and stirred at room temperature overnight to construct GP-AuNPs@Ce6. Of note, these excess free or unreacted Ce6 was removed by centrifugation $(15000 \mathrm{rpm}, 20 \mathrm{~min})$ for over three times. At last, the GPAuNPs@Ce6, the $9.0 \mathrm{mg}$ of NHS-diazirine was added into above prepared solutions, after stirring for 2-3 $\mathrm{h}$ at room temperature, the reaction mixture was subjected to centrifugal ( $15000 \mathrm{rpm}, 15 \mathrm{~min}$ ) for three times to afford the desired GP-dAuNPs@Ce6. Then the product of GP-dAuNPs@Ce6 was collected and stored at $4{ }^{\circ} \mathrm{C}$ in the dark for the following experiments. The morphology and size of as-prepared nanoprobes were examined by transmission electronic microscopy (TEM, Philips CM 200) with the 200kV. The UV-vis absorption spectra were measured by a 750 UV-vis near-infrared spectrophotometer (PerkinElmer lambda). The photoluminescence (PL) spectra were recorded by a spectro-fluorimeter (HORIBA JOBIN YVON FLUORMAX-4). The dynamic light scattering (DLS) and Zeta potential was analyzed by the Delsa $^{\text {TM }}$ nano submicron particle size and Zeta potential particle analyzer (Beckman Coulter, Inc.).

\section{Bacterial culture.}

Escherichia coli (EC) (ATCC 11303) were purchased from American type culture collection (ATCC). Staphylococcus aureus (SA) were obtained from the First Affiliated Hospital of Soochow University. Micrococcus luteus (ML) (BNCC 102589) and Pseudomonas aeruginosa (PA) (BNCC 125486) were purchased from BeNa Culture Collection (BNCC, Shanghai, China). All bacterial culture reagents (e.g., LB medium, etc.) were obtained from Sangon Biotech (Shanghai) Co., Ltd. The lyophilized powder of four kinds of bacteria was dissolved in LB medium. The bacteria liquid was coated on LB plate medium and cultured in $37^{\circ} \mathrm{C}$ incubator for $12 \mathrm{~h}$. After that, a single colony was picked from the plate and cultured in LB liquid medium for $12 \mathrm{~h}$. And then bacterial cells were grown in LB medium at $250 \mathrm{rpm}$ and $37^{\circ} \mathrm{C}$ and obtained at the exponential growth phase. Finally, the bacterial suspensions were washed twice and resuspended in PBS buffer for the next use. The concentration of bacteria was detected by measuring the 
optical density (OD) at $600 \mathrm{~nm}$. The number of bacterial colonies was counted by a colony counting instrument (Czone 8).

\section{In vitro imaging of bacteria.}

The $20 \mu \mathrm{L}$ of purified and re-suspended bacterial suspension $\left(1.0 \times 10^{7} \mathrm{CFU}\right)$ was incubated with GPdAuNPs@Ce6 $\left(1.0 \mathrm{mg} \mathrm{mL}^{-1}, 200 \mu \mathrm{L}\right)$ for $2 \mathrm{~h}$ in a shaking incubator $(200 \mathrm{rpm})$ at $37^{\circ} \mathrm{C}$. The bacteria were harvested by centrifuging the mixture at $6000 \mathrm{rpm}$ for $5 \mathrm{~min}$ in Eppendorf (EP) tubes. The resulting bacteria were re-suspended and washed with PBS for three times. Then $10 \mu \mathrm{L}$ of the washed bacteria solution was transferred onto a microscope slide covered by a coverslip, and then imaged by a confocal laser scanning microscope (CLSM, Leica, TCSSP5 II) with 30\% power of diode laser. All fluorescence images were captured by CLSM with a $\times 64$ oil-immersion objective and taken under the same optical conditions, and the same brightness and contrast was applied to the images by the microscope automatically. The processing and analysis of ROI was performed by the commercial image analysis software (Leica Application Suite Advanced Fluorescence Lite). Moreover, the distribution of GPdAuNPs@Ce6 in the bacterial cells were confirmed by TEM (Philips CM 200).

\section{In vivo imaging of bacteria.}

All in vivo experiments were performed on female nude mice (SPF grade, 6-8 weeks old), under a protocol approved by the animal care committee of Soochow University. To construct the bacteriainfected mice model, the mice were anesthetized by intraperitoneal injection of $125 \mu \mathrm{L}$ of $1 \%$ Pentobarbital Sodium. And then we subcutaneously injected $50 \mu \mathrm{L}$ of $\sim 1.1 \times 10^{7}$ CFU SA or $\sim 0.8 \times 10^{7}$ CFU PA into the left or right caudal thigh of the mice. To determine the final number of bacteria at the infection sites during imaging, the infected tissues were harvested, followed by homogenization in the 1 $\mathrm{mL}$ of sterile PBS buffer. Next, we collected the bacteria suspension by centrifugation at $1000 \mathrm{rpm}$ to remove tissue fragments. Finally, we diluted the collected bacteria with PBS buffer and cultured them on an agarose medium at $37^{\circ} \mathrm{C}$ for $12 \mathrm{~h}$. We used a colony counting instrument (Czone 8 ) to count the bacterial colonies. In this case, the final concentration of SA or PA at the infection site during imaging is $\sim 1.0 \times 10^{7} \mathrm{CFU}$. On the other aspect, we subcutaneously injected $50 \mu \mathrm{L}$ of PBS or $\sim 0.6 \times 10^{7} \mathrm{CFU} \mathrm{PA}+$ $S A$ into the left and right caudal thigh of the mice. By using the same CFU counting method, the final concentration of $\mathrm{PA}+\mathrm{SA}$ at the infection site during imaging is $\sim 1.0 \times 10^{7} \mathrm{CFU}$. To assess the limit of detection, we subcutaneously injected $50 \mu \mathrm{L}$ of $\sim 1.3 \times 10^{5} \mathrm{CFU}$ SA or $\sim 1.1 \times 10^{5} \mathrm{CFU}$ PA. Also, the final amount of SA or PA at the infection site during imaging is $\sim 1.0 \times 10^{5} \mathrm{CFU}$ by using the same CFU counting method. Afterwards, we intravenously injected GP-dAuNPs@Ce6 $\left(1.0 \mathrm{mg} \mathrm{mL}^{-1}, 100 \mu \mathrm{L}\right)$ into the infected mice. After 24-h injection, we imaged the treated mice by using an in vivo optical imaging system (IVIS Lumina III) $\left(\lambda_{\mathrm{ex}}=460 \mathrm{~nm}, \lambda_{\mathrm{em}}=670 \mathrm{~nm}\right)$ or a Vevo2100 LAZR imaging system (Vevo®LAZR, VisualSonics, Inc., Canada) with PA-mode.

To construct tumour containing bacteria model, we subcutaneously injected $100 \mu \mathrm{L}$ of $4 \mathrm{~T} 1$ cell suspension into the right back region of nude mice. When the tumour size was up to $\sim 100 \mathrm{~mm}^{3}$, the mice 
were randomly divided into two groups. In one group, we subcutaneously injected bacteria into the left thigh (SA: $50 \mu \mathrm{L}, \sim 1.1 \times 10^{7} \mathrm{CFU}$. PA: $50 \mu \mathrm{L}, \sim 0.8 \times 10^{7} \mathrm{CFU}$ ), but not into the right tumour. In the other group, we subcutaneously injected bacteria into the left thigh (SA: $50 \mu \mathrm{L}, \sim 1.1 \times 10^{7}$ CFU. PA: $50 \mu \mathrm{L}, \sim 0.8$ $\times 10^{7} \mathrm{CFU}$ ) as well as the right tumour (SA: $50 \mu \mathrm{L}, \sim 1.1 \times 10^{7} \mathrm{CFU}$. PA: $50 \mu \mathrm{L}, \sim 0.8 \times 10^{7} \mathrm{CFU}$ ). The actual number of bacteria at the infection sites during imaging was also determined via tissue harvesting, homogenization and culturing with CFU count. The final concentration of SA or PA at the infection site during imaging is $1.0 \times 10^{7}$ CFU. Next, we intravenously injected GP-dAuNPs@Ce6 $\left(1.0 \mathrm{mg} \mathrm{mL}^{-1}, 100\right.$ $\mu \mathrm{L}$ ) into the infected mice. After $24 \mathrm{~h}$, we imaged the treated mice by an in vivo optical imaging system (IVIS Lumina III) $\left(\lambda_{\mathrm{ex}}=460 \mathrm{~nm}, \lambda_{\mathrm{em}}=670 \mathrm{~nm}\right)$ or a Vevo2100 LAZR imaging system (Vevo®LAZR, VisualSonics, Inc., Canada) with PA-mode. In order to image the bacteria in the tumour or infection site, we placed the mice in a prone position, and positioned the photoacoustic probe in the tumour or infection site.

To construct gastrointestinal tract containing bacteria model, we injected the agarose gel containing E.coli $(E C)$ into the gut lumen of the Balb/c nude mice (female, 6-7 weeks old). The final concentration of $\mathrm{EC}$ was $\sim 10^{7} \mathrm{CFU}$, which was determined by tissue harvesting, homogenization and culturing with $\mathrm{CFU}$ count as mentioned above. Afterwards, we intravenously injected GP-dAuNPs@Ce6 (1.0 mg mL ${ }^{-1}, 100$ $\mu \mathrm{L}$ ) into the infected mice. At 24-h postinjection of GP-dAuNPs@Ce6, we imaged the gut by an in vivo optical imaging system (IVIS Lumina III) $\left(\lambda_{\mathrm{ex}}=460 \mathrm{~nm}, \lambda_{\mathrm{em}}=670 \mathrm{~nm}\right)$ or a Vevo2100 LAZR imaging system (Vevo®LAZR, VisualSonics, Inc., Canada) with PA-mode. For photoacoustic imaging of $E$. coli in the gut, we placed the mice in a supine position, and positioned the photoacoustic probe above the lower abdomen, transverse to the gut.

\section{In vitro antibacterial assays.}

SA or EC were respectively treated by PBS, AuNPs, dAuNPs@Ce6, GP-AuNPs@Ce6 and GP-dAuNPs@Ce6, followed by different irradiations of 405, 660 and $808 \mathrm{~nm}$ laser (405-nm laser:1.0 W cm ${ }^{-2}, 25 \mathrm{~min}$; 660$\mathrm{nm}$ laser:12 mW cm $\mathrm{m}^{-2}, 5 \mathrm{~min}$; 808-nm laser:1.0 $\mathrm{W} \mathrm{cm}^{-2}, 5 \mathrm{~min}$ ). We characterized the morphology of bacteria after treatment by using SEM (FEI Quanta 200F). The antibacterial rate was calculated based on the bacteria colonies on the agar plates. The antibacterial rate was obtained according to Eq. (1):

Antibacterial rate $(\%)=\left(N_{\text {control }}-N_{\text {experiment }}\right) / N_{\text {control }} \times 100 \%(1)$,

where " $N_{\text {control }}$ and " $N_{\text {experiment" }}$ " respectively stand for bacterial counts (CFU mL ${ }^{-1}$ ) from the control groups of "PBS" and other experiment groups (experiment).

In vivo antibacterial assays. To asess the antibacterial ability of Pac-Man strategy in vivo, $50 \mu \mathrm{L}$ of SA ( $\left.1.1 \times 10^{7} \mathrm{CFU}\right)$ or PA $\left(\sim 0.8 \times 10^{7} \mathrm{CFU}\right)$ were subcutaneously injected into the right thigh of the mice, respectively. Then, these infected mice were intravenously injected with $100 \mu \mathrm{L}$ of $1.0 \mathrm{mg} \mathrm{mL}^{-1} \mathrm{GP}$ dAuNPs@Ce6 respectively. The bacterial cell concentration was $10^{7}$ CFU during treatment, which was determined via tissue harvesting, homogenization and culturing with CFU count. Then these mice were 
divided into six groups: the mice in group 1 (G1) are treated with GP-dAuNPs@Ce6 + 660-nm laser (12 $\mathrm{mW} \mathrm{cm}{ }^{-2}, 5 \mathrm{~min}$ ); the mice in group 2 (G2) are treated with GP-dAuNPs@Ce6 + 808-nm laser $\left(1.0 \mathrm{~W} \mathrm{~cm}^{-2}\right.$, $5 \mathrm{~min}$ ); the mice in group 3 (G3) are treated by GP-dAuNPs@Ce6 +660-nm laser (12 mW cm $\left.{ }^{-2}, 5 \mathrm{~min}\right)+$ 808-nm laser (1.0 W cm - $\left.^{-}, 5 \mathrm{~min}\right)$; the mice in group 4 (G4) are treated by GP-dAuNPs@Ce6 + 405-nm laser $\left(1.0 \mathrm{~W} \mathrm{~cm}^{-2}, 25 \mathrm{~min}\right)+660-\mathrm{nm}$ laser $\left(12 \mathrm{~mW} \mathrm{~cm}^{-2}, 5 \mathrm{~min}\right)$; the mice in group 5 (G5) are treated by GP-dAuNPs@Ce6 + 405-nm laser (1.0 W cm $\left.{ }^{-2}, 25 \mathrm{~min}\right)+808-\mathrm{nm}$ laser $\left(1.0 \mathrm{~W} \mathrm{~cm}^{-2}, 5 \mathrm{~min}\right)$; the mice in group 6 (G6) are treated by the GP-dAuNPs@Ce6 + 405-nm laser (1.0 W cm $\left.{ }^{-2}, 25 \mathrm{~min}\right)+660$-nm laser (12 $\left.\mathrm{mW} \mathrm{cm}{ }^{-2}, 5 \mathrm{~min}\right)+808-\mathrm{nm}$ laser $\left(1.0 \mathrm{~W} \mathrm{~cm}^{-2}, 5 \mathrm{~min}\right)$. The wound area was photographed and the size of wound area was processed by ImageJ software. At the last day of treatment, we extracted the bacteria from the infected tissues of the mice $(n=3)$ and cultured them on the agar plates. We counted the final bacteria colonies for calculating the corresponding antibacterial rate according to Eq. (1). Meanwhile, the infected tissues from each group of mice were fixed in the 4\% PFA solution for the following H\&E, Masson or Gram staining.

\section{In vitro and in vivo toxicity assessment.}

Human cervical cells (HeLa cells), human embryonic kidney 293T cells (HEK-293T cells), monkey embryo kidney epithelial cells (Marc-145 cells), Human normal embryonic liver cells (LO2 cells) and human breast cancer cells (MCF-7 cells), cultured in the Dulbecco's modified Eagle's medium with high glucose $(\mathrm{H}-$ DMEM), were purchased from Shanghai Zhong Qiao Xin Zhou Biotechnology Co., Ltd (China). All abovementioned media were supplemented with $10 \%$ heat-inactivated fetal bovine serum (FBS) and $1 \%$ relevant antibiotics $\left(100 \mu \mathrm{g} \mathrm{mL}^{-1}\right.$ streptomycin and $100 \mathrm{U} \mathrm{mL}^{-1}$ penicillin). All cell lines were cultured at $37^{\circ} \mathrm{C}$ in a $5 \% \mathrm{CO}_{2}$ incubator with the humidified atmosphere. We used the established colorimetric MTT assay to evaluate the cytotoxicity of GP-dAuNPs@Ce6. Typically, L02, HEK-293T, Marc-145, HeLa or MCF7 cells were seeded into the 96 -well cell culture plate at a density of $\sim 1.0-1.5 \times 10^{4} /$ well for $24 \mathrm{~h}\left(37^{\circ} \mathrm{C}\right.$, $\left.5 \% \mathrm{CO}_{2}\right)$, followed by the treatment of GP-dAuNPs@Ce6 with different concentrations $(0,2,1,0.5,0.25$, $0.125 \mathrm{mg} \mathrm{mL}^{-1}$ ), respectively. The $20 \mu \mathrm{L}$ of $5 \mathrm{mg} \mathrm{mL}^{-1} \mathrm{MTT}$ was added into each well for another incubation with cells for $6 \mathrm{~h}$ at $37^{\circ} \mathrm{C}$. Afterwards, these treated cells were lysed by using the acidified sodium dodecyl sulfate (SDS) $(100 \mu \mathrm{L} /$ well). We determined the cell viability by the measurement of the absorbance at $570 \mathrm{~nm}$ via a microplate reader (BioRad 680, USA). On the other aspect, we used the staining of tissue sections to assess the biocompatibility of GP-dAuNPs@Ce6 in vivo. Specifically, we harvested the main organs (e.g., heart, liver, spleen, lung, kidney, and brain) from the mice after 30-day treatment of $100 \mu \mathrm{L}$ of GP-dAuNPs@Ce6 $(1.0 \mathrm{mg} / \mathrm{mL})$ and various irradiations. The collected organs were fixed by $4 \%$ PFA solution, mounted with paraffin, and sliced, followed by hematoxylin and eosin $(\mathrm{H}$ \& E) staining, Masson's trichrome staining, and Gram staining.

\section{Statistical analysis.}

For statistical significance testing, we used a one-way ANOVA analysis or the paired two-tailed t-test (* means $p<0.05$, ** means $p<0.01$, *** means $p<0.001$, **** means $p<0.0001$, ns means no 
significance). The statistical analysis was performed by using the software of Origin or GraphPad Prism. Error bars represent the standard deviation obtained from three independent measurements. All imaging experiments were repeated three times with similar results. Region of interest (ROI) was employed for quantitative assessments of fluorescence intensity, which was calculated by the commercial image analysis software (Leica Application Suite Advanced Fluorescence Lite, LAS AF Lite) and the software of ImageJ (NIH Image; http;//rsbweb.nih.gov/ij/).

\section{Declarations}

Life Science Reporting Summary. Further information on experimental design is available in the Life Science Reporting Summary.

Data availability. The data that support the findings of this study are available within the paper and its supplementary information.

\section{Acknowledgements}

We thank Prof. Shuit-Tong Lee, Dr. Liangzhu Feng (Soochow University, China) and Dr. Fei Peng (Harvard University, USA) for their general help and valuable suggestions. The authors acknowledge financial support from National Natural Science Foundation of China (No. 21825402 and 22074101), Natural Science Foundation of Jiangsu Province of China (No. BK20191417) and the Program for Jiangsu Specially-Appointed Professors to the Prof. Yao He, a project funded by the Priority Academic Program Development of Jiangsu Higher Education Institutions (PAPD), 111 Project as well as the Collaborative Innovation Center of Suzhou Nano Science and Technology (NANO-CIC).

\section{Author Contributions}

Y. M. Y., B. B. C., H. Y. W. and Y. H. conceived and designed the research. Y. M. Y. and B. B. C. carried out most of experiments and analyzed the data. Y. M. Y. B. B. C., J. Y. C., J. L. T. and B. S performed additional experiments and characterizations. Y. M. Y., B. B. C., H. Y. W and Y. H wrote the manuscript.

\section{Competing interests}

The authors declare no competing financial interests.

\section{Additional information}

Supplementary Information is available in the online version of the paper.

Reprints and permissions information is available online at www.nature.com/reprints.

Correspondence and requests for materials should be addressed to H. Y. W. or Y. H. 
Publisher's note: Springer Nature remains neutral with regard to jurisdictional claims in published maps and institutional affiliations.

\section{References}

1. Bourdeau, R., Lee-Gosselin, A., Lakshmanan, A. et al. Acoustic reporter genes for noninvasive imaging of microorganisms in mammalian hosts. Nature 553, 86-90 (2018).

2. Geller, L. T., Barzily-Rokni, M., Danino, D. et al. Potential role of intratumor bacteria in mediating tumor resistance to the chemotherapeutic drug gemcitabine. Science. 357, 1156-1160 (2017).

3. Nejman, D., Livyatan, I., Fuks, G. et al. The human tumor microbiome is composed of tumor typespecific intracellular bacteria. Science. 368, 973-980 (2020).

4. Donaldson, G., Lee, S. \& Mazmanian, S. Gut biogeography of the bacterial microbiota. Nat. Rev. Microbiol. 14, 20-32 (2016).

5. Round, J., Mazmanian, S. The gut microbiota shapes intestinal immune responses during health and disease. Nat. Rev. Immunol. 9, 313-323 (2009).

6. Wang, Y., Kasper, L. H. The role of microbiome in central nervous system disorders. Brain Behav. Immun. 38, 1-12 (2014).

7. Derrien, M., Vlieg, J. E. T. v. H. Fate, activity, and impact of ingested bacteria within the human gut microbiota. Trends Microbiol. 23, 354-366 (2015).

8. Matson, V., Fessler, J., Bao, R. et al. The commensal microbiome is associated with anti-PD-1 efficacy in metastatic melanoma patients. Science. 359, 104-108 (2018).

9. Routy, B., Chatelier, E. L., Derosa, L. et al. Gut microbiome influences efficacy of PD-1-based immunotherapy against epithelial tumors. Science 359, 91-97 (2018).

10. Din, M., Danino, T., Prindle, A. et al. Synchronized cycles of bacterial lysis for in vivo delivery. Nature 536, 81-85 (2016).

11. Danino, T., Prindle, A., Kwong, G. A. et al. Programmable probiotics for detection of cancer in urine. Sci. Transl. Med. 7, 289 ra284 (2015).

12. Riglar, D., Giessen, T., Baym, M. et al. Engineered bacteria can function in the mammalian gut longterm as live diagnostics of inflammation. Nat. Biotechnol. 35, 653-658 (2017).

13. Poore, G.D., Kopylova, E., Zhu, Q. et al. Microbiome analyses of blood and tissues suggest cancer diagnostic approach. Nature 579, 567-574 (2020).

14. Errico, C., Pierre, J., Pezet, S. et al. Ultrafast ultrasound localization microscopy for deep superresolution vascular imaging. Nature 527, 499-502 (2015).

15. Bertolotti, J., van Putten, E., Blum, C. et al. Non-invasive imaging through opaque scattering layers. Nature 491, 232-234 (2012).

16. Katz, O., Heidmann, P., Fink, M. et al. Non-invasive single-shot imaging through scattering layers and around corners via speckle correlations. Nat. Photon. 8, 784-790 (2014). 
17. Papadopoulos, I. N., Jouhanneau, J. S., Poulet, J. F. A. et al. Scattering compensation by focus scanning holographic aberration probing (F-SHARP). Nat. Photon. 11, 116-123 (2017).

18. Imai, Y., Meyer, K.J., linishi, A. et al. A new antibiotic selectively kills Gram-negative pathogens. Nature 576, 459-464 (2019).

19. Sousa, M. C. New antibiotics target the outer membrane of bacteria. Nature 576, 389-390 (2019).

20. Hou, X., Zhang, X., Zhao, W. et al. Vitamin lipid nanoparticles enable adoptive macrophage transfer for the treatment of multidrug-resistant bacterial sepsis. Nat. Nanotechnol. 15, 41-46 (2020).

21. Fries, C. N., Curvino, E. J., Chen, J. L. et al. Advances in nanomaterial vaccine strategies to address infectious diseases impacting global health. Nat. Nanotechnol. (2020).

22. Wang, L. V., Hu, S. Photoacoustic tomography: in vivo imaging from organelles to organs. Science. $335,1458-1462(2018)$

23. Ruan, H., Liu, Y., Xu, J. et al. Fluorescence imaging through dynamic scattering media with speckleencoded ultrasound-modulated light correlation. Nat. Photonics. 14, 511-516 (2020).

24. Arvizo, R. R. Bhattacharyya, S., Kudgus, R. A. et al. Intrinsic therapeutic applications of noble metal nanoparticles: past, present and future. Chem. Soc. Rev. 41, 2943-2970 (2012).

25. Doane, T. Burda,C. The unique role of nanoparticles in nanomedicine: imaging, drug delivery and therapy. Chem. Soc. Rev. 41, 2885-2911 (2012).

26. Qin, Z. Bischof, J. C. Thermophysical and biological responses of gold nanoparticle laser heating. Chem. Soc. Rev. 41, 1191-1217 (2012).

27. Xia, H., Gao, Y., Yin, L. et al. Light-triggered covalent coupling of gold nanoparticles for photothermal cancer therapy. ChemBioChem 20,667-671, (2019).

28. Cheng, X., Sun, R., Yin, L. et al. Photothermal therapy: light-triggered assembly of gold nanoparticles for photothermal therapy and photoacoustic imaging of tumors in vivo. Adv. Mater. 291604894 (2017).

29. Deng, H., Dai, F., Ma, G. et al. Theranostic gold nanomicelles made from biocompatible comb-like polymers for thermochemotherapy and multifunctional imaging with rapid clearance. Adv. Mater. 27, 3645-3653 (2015).

30. Canton, I., Battaglia, G. Endocytosis at the nanoscale. Chem. Soc. Rev. 41, 2718-2739 (2012).

31. Dykman, L. A., Khlebtsov, N. G. Uptake of engineered gold nanoparticles into mammalian Cells. Chem. Rev. 114, 1258-1288 (2014).

32. Behzadi, S., Serpooshan, V., Tao, W. et al. Cellular uptake of nanoparticles: journey inside the cell. Chem. Soc. Rev. 46, 4218-4244 (2017).

33. Ning, X., Lee, S., Wang, Z. et al. Maltodextrin-based imaging probes detect bacteria in vivo with high sensitivity and specificity. Nat. Mater. 10, 602-607 (2011).

34. Zlitni, A., Gowrishankar, G., Steinberg, I. et al. Maltotriose-based probes for fluorescence and photoacoustic imaging of bacterial infections. Nat. Commun. 11, 1250 (2020). 
35. Ning, X., Seo, W., Lee, S. et al. PET imaging of bacterial infections with fluorine-18-labeled maltohexaose. Angew. Chem. Int. Ed. 53, 14096-14101 (2014).

36. Takemiya, K., Ning, X., Seo, W. et al. Novel PET and near infrared imaging probes for the specific detection of bacterial infections associated with cardiac devices. JACC-Cardiovasc. Imag. 12, 875886 (2019).

37. Pang, X., Xiao, Q., Cheng, Y. et al. Bacteria-responsive nanoliposomes as smart sonotheranostics for multidrug resistant bacterial infections. ACS Nano. 13, 2427-2438 (2019).

38. Bordignon, E., Grote, M. \& Schneider, E. The maltose ATP-binding cassette transporter in the 21st century - towards a structural dynamic perspective on its mode of action Mol. Microbiol. 77, 13541366 (2010).

39. Shuman, H. A. The maltose-maltodextrin transport system of Escherichia coli. Ann. Microbiol. 133A, 153-159 (1982).

40. Klebba, P. E. Mechanism of maltodextrin transport through LamB. Res. Microbiol. 153, 417-424 (2002).

41. Charbit, A. Maltodextrin transport through LamB. Front. Biosci. 8, s265-274 (2003).

42. Boos, W., Shuman, H. Maltose/maltodextrin system of Escherichia coli: transport, metabolism, and regulation. Microbiol. Mol. Biol. Rev. 62, 204-229 (1998).

43. Dippel, R., Boos, W. The maltodextrin system of Escherichia coli: metabolism and transport. J. Bacteriol. 187, 8322-8331 (2005).

44. Freundlieb, S., Ehmann, U. \& Boos, W. Facilitated diffusion of p-nitrophenyl-alpha-D-maltohexaoside through the outer membrane of Escherichia coli. Characterization of LamB as a specific and saturable channel for maltooligosaccharides. J. Biol. Chem. 263, 314-320 (1988).

45. Gopal, S., Daniela, B., Nicole, H. et al. Maltose and maltodextrin utilization by Listeria monocytogenes depend on an inducible ABC transporter which is repressed by glucose. PLoS One 5, e10349 (2010).

46. Schonert, S., Seitz, S., Krafft, H. et al. Maltose and maltodextrin utilization by Bacillus subtilis. J. Bacteriol. 188, 3911-3922 (2006).

47. Morbach, S., Tebbe, S. \& Schneider, E. The ATP-binding cassette (ABC) transporter for maltose/maltodextrins of Salmonella typhimurium. Characterization of the ATPase activity associated with the purified Malk subunit. J. Biol. Chem. 268, 18617-18621 (1993).

48. Blencowe, A., Hayes, W. Development and application of diazirines in biological and synthetic macromolecular systems. Soft Matter 1, 178-205 (2005).

49. Wollman, E. W., Kang, D., Frisbie, C. D. et al. Photosensitive self-assembled monolayers on gold: photochemistry of surface-confined aryl azide and cyclopentadienylmanganese tricarbonyl. J. Am. Chem. Soc. 116, 4395-4404 (1994).

50. Ismaili, H., Lee, S. \& Workentin, M. S. Diazirine-modified gold nanoparticle: template for efficient photoinduced interfacial carbene insertion reactions. Langmuir 26, 14958-14964 (2010). 
51. Tang, J., Chu, B., Wang, J. et al. Multifunctional nanoagents for ultrasensitive imaging and photoactive killing of Gram-negative and Gram-positive bacteria. Nat. Commun. 10, 4057 (2019).

52. Leary, S. C., Hill, B. C., Lyons, C. N. et al. Chronic treatment with azide in situ leads to an irreversible loss of cytochrome c oxidase activity via holoenzyme dissociation. J. Biol. Chem. 277, 11321-11328 (2002).

53. van Oosten, M., Schäfer, T., Gazendam, J. et al. Real-time in vivo imaging of invasive- and biomaterial-associated bacterial infections using fluorescently labelled vancomycin. Nat. Commun. 4, 2584 (2013).

54. van Oosten M., Hahn, M., Crane, L. M. A. et al. Targeted imaging of bacterial infections: advances, hurdles and hopes. FEMS Microbiol. Rev. 39, 892-916 (2015).

55. Ding, Y. F., Li, S., Liang, L. et al. Highly biocompatible chlorin e6-loaded chitosan nanoparticles for improved photodynamic cancer therapy. ACS Appl. Mater. Interfaces 10, 9980-9987 (2018).

56. Feng, L., Cheng, L., Dong, Z. et al. Theranostic liposomes with hypoxia-activated prodrug to effectively destruct hypoxic tumors post-photodynamic therapy. ACS Nano 11, 927-937 (2017).

57. Dubinsky, L., Krom, B. P., Meijler, M. M. et al. Diazirine based photoaffinity labeling. Bioorgan. Med. Cham. 20, 554-570, (2012).

58. Shi, H., Zhang, C. J., Chen, G. Y. J. et al. Cell-Based Proteome Profiling of Potential Dasatinib Targets by Use of Affinity-Based Probes. J. Am. Chem. Soc. 134, 3001-3014.

59. Hernandez, F. J., Huang, L., Olson, M. E. et al. Non-invasive imaging of Staphylococcus aureus infections with a nuclease-activated probe. Nat. Med. 20, 301-306 (2014).

60. Akram, A. R., Avlonitis, N., Lilienkampf, A. et al. A labelled-ubiquicidin antimicrobial peptide for immediate in situ optical detection of live bacteria in human alveolar lung tissue. Chem. Sci. 6, 6971-6979 (2015).

61. Li, L. L., Ma, H. L., Qi, G. B. et al. Pathological condition driven construction of supramolecular nanoassemblies for bacterial infection detection. Adv. Mater. 28, 254-262 (2016).

62. Gorbach, S. L. in Medical Microbiology 4th edn (ed. Baron, S.) Ch. 95 (Univ. Texas Medical Branch, 1996).

63. Sonnenborn, U. \& Schulze, J. The non-pathogenic Escherichia coli strain Nissle 1917-features of a versatile probiotic. Microb. Ecol. Health Dis. 21, 122-158 (2009).

\section{Figures}




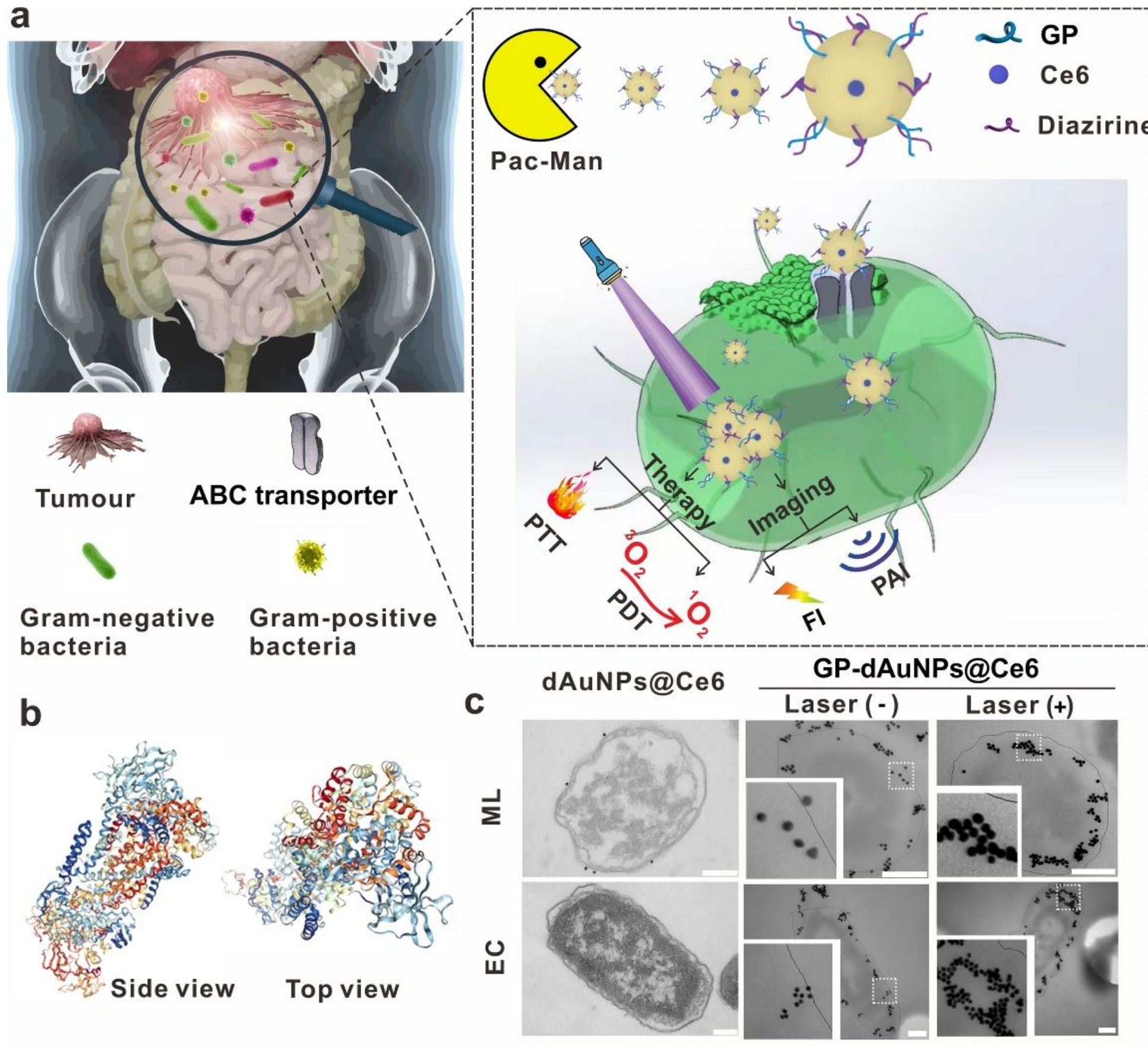

Figure 1

Schematic design and characterization of Pac-Man bacteria eating gold nanoparticles for aggregationenhanced imaging and killing bacteria. a, Schematic showing Pac-Man bacteria eating gold nanoparticles for aggregation-enhanced imaging and killing bacteria. The imaging modals include fluorescence imaging $(\mathrm{FI})$ and photoacoustic imaging (PAI). The therapeutic methods include photothermal therapy (PTT) and photodynamic therapy (PDT). b, Structure of ABC transporter in E. coli (Left: side view; Right: top view). The sequences of ABC transporter in E. coli were obtained from http://www.rcsb.org/ and the structure was simulated by PyMOL software. c. TEM images of M. luteus $(\mathrm{ML})$ or E. coli (EC) treated by $1.0 \mathrm{mg} \mathrm{mL}-1$ of diazirine and chlorin e6 (Ce6)-modified gold nanoparticles (AuNPs) (dAuNPs@Ce6) or GP (e.g, poly[4-0-(a-D-glucopyranosyl)-D-glucopyranose]), diazirine and Ce6- 
modified gold nanoparticles (AuNPs) (GP-dAuNPs@Ce6) at 37 oC for $2 \mathrm{~h}$. After incubation, the treated bacteria were rinsed with PBS buffer for several times. GP-dAuNPs@Ce6-treated bacteria were subjected with or without laser irradiation ( $405 \mathrm{~nm}, 1.0 \mathrm{~W} \mathrm{~cm}-2,25 \mathrm{~min}$ ). The bacterial cell concentration is $\sim 1.0$ $\times 107 \mathrm{CFU}$. Scale bars, $200 \mathrm{~nm}$. All imaging experiments were repeated three times with similar results. The cartoons are created by Dr. Houyu Wang

a
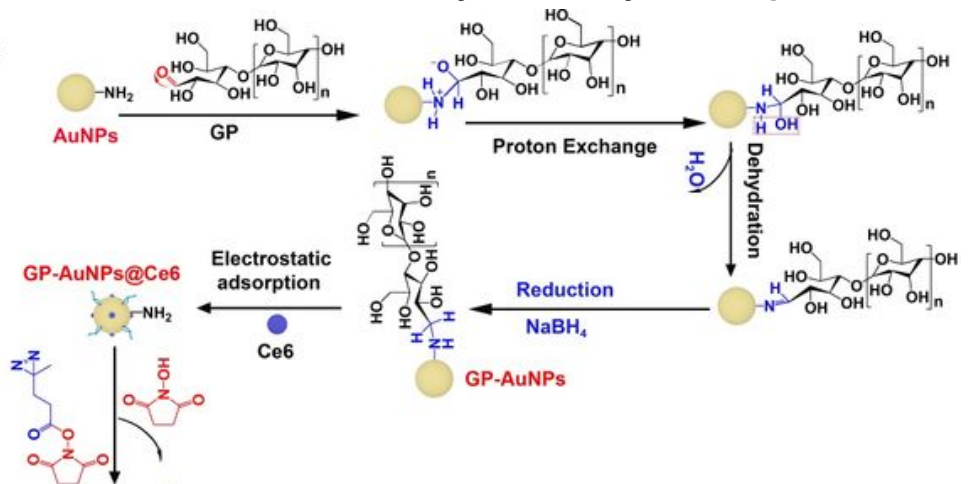

GP-AuNPs
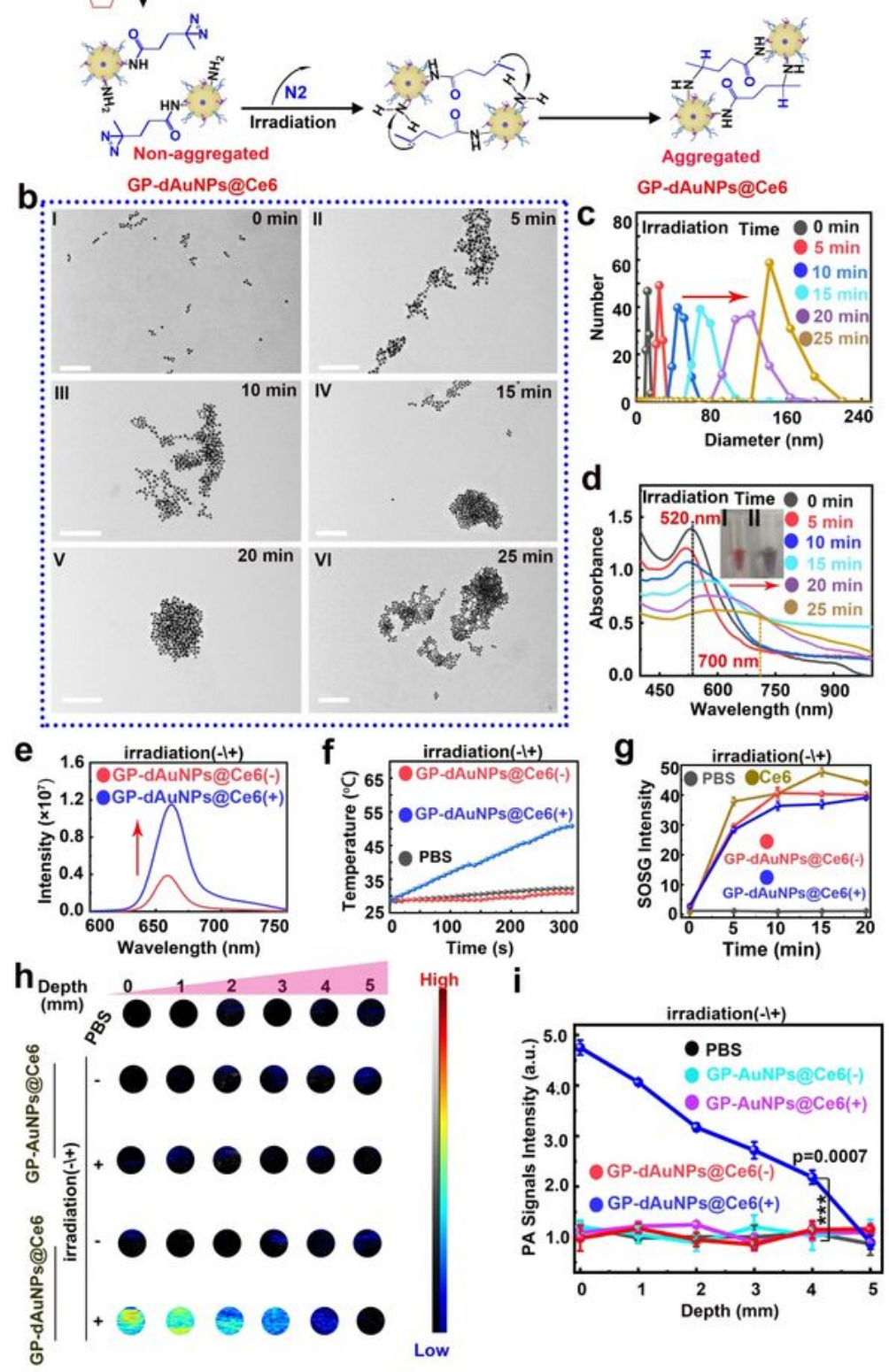

Figure 2 
Schematic and characterization of nanoprobes of GP-dAuNPs@Ce6. a, Schematic illustrating the synthesis of GP, diazirine and Ce6-modified gold nanoparticles (AuNPs) (GP-dAuNPs@Ce6) step by step and the mechanism of the formation of aggregated GP-dAuNPs@Ce6 under laser irradiation. b, Irradiation time-dependent TEM images of GP-dAuNPs@Ce6 (405 nm, 1.0 W cm-2). Scale bars, 200 nm. c, Corresponding irradiation time-dependent hydrodynamic size profiles of GP-dAuNPs@Ce6 (405 nm, 1.0 W cm-2).d, Corresponding irradiation time-dependent absorption spectra of GP-dAuNPs@Ce6 (405 nm, 1.0 W cm-2). e, PL spectra of 0.5 mg mL-1 GP-dAuNPs@Ce6 with (+) and without laser irradiation (-). $f$, The photothermal heating curves of PBS, non-aggregated GP-dAuNPs@Ce6 and aggregated GP-dAuNPs@Ce6 under the irradiation of 808-nm laser. g, Evaluation of 102 generation by PBS, free Ce6, non-aggregated GP-dAuNPs@Ce6 and aggregated GP-dAuNPs@Ce6 by using the SOSG assay. h, Chicken breast tissue thickness-dependent photoacoustic signals of 1.0 mg mL-1 GP-dAuNPs@Ce6 or GP-dAuNPs@Ce6 with $(+)$ and without laser irradiation (-), i, Corresponding plots of photoacoustic intensity versus chicken breast tissue thickness. All imaging experiments were repeated three times with similar results. Statistical analysis was performed using a one-way ANOVA analysis. Error bars represent the standard deviation obtained from three independent measurements ( $* \star \star$ means $p<0.001, n=3$ ). Source data are provided as a Source Data file 


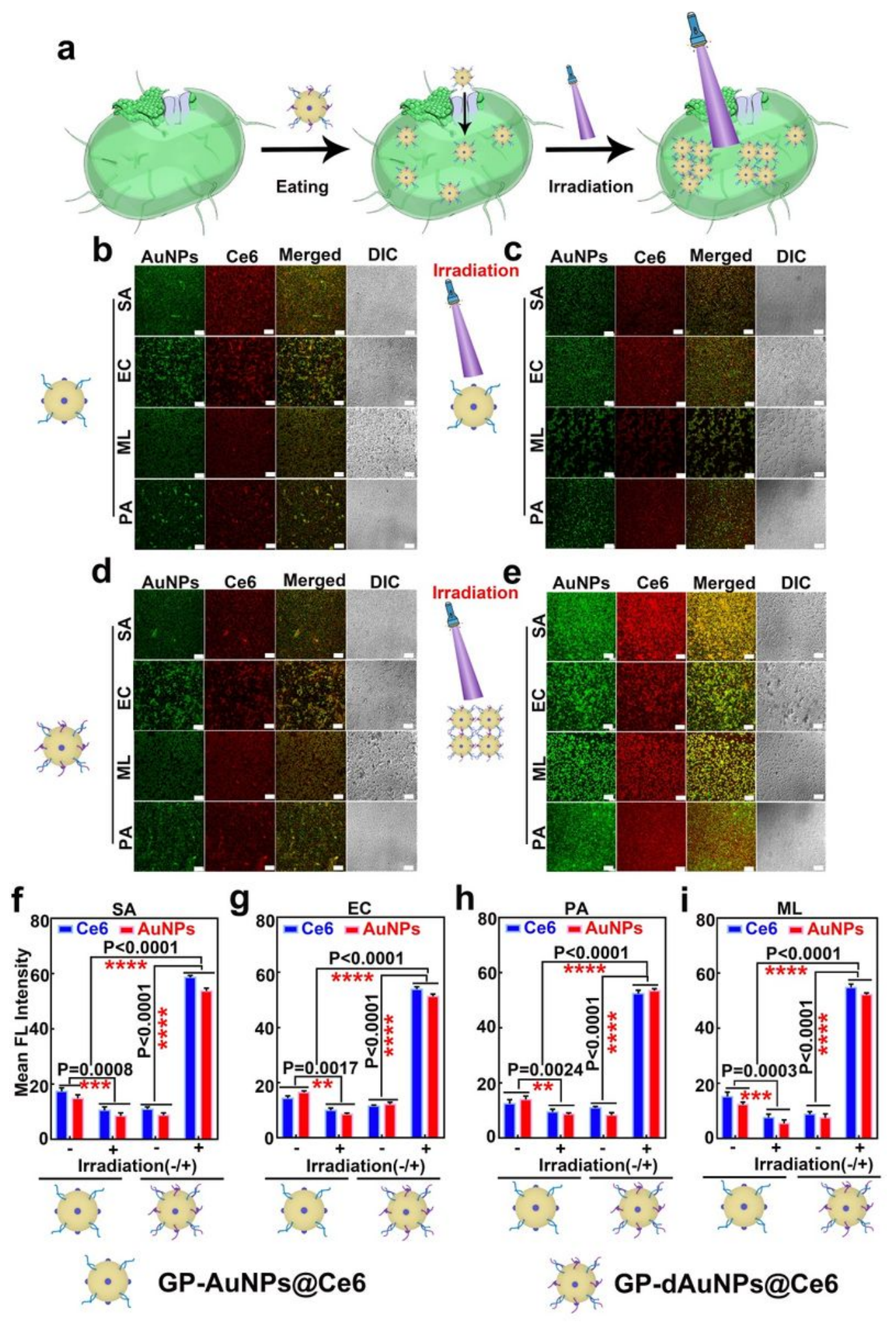

Figure 3

In vitro imaging of Gram-negative and Gram-positive bacteria based on the proposed Pac-Man strategy. a, Schematic illustrating Pac-Man bacteria eating nanoprobes for in vitro fluorescent imaging. b-e, CLSM images of four different kinds of bacteria (S. aureus (SA), E. coli (EC), M. luteus (ML), P. aeruginosa (PA)) incubated with 1.0 mg mL-1 GP-AuNPs@Ce6 without (b) or with (c) 405-nm laser irradiation, or GPdAuNPs@Ce6 without (d) or with (e) 405-nm laser irradiation. After incubation, the treated bacteria were 
rinsed with PBS buffer for several times. The bacterial cell concentration is $\sim 1.0 \times 107 \mathrm{CFU}$. Laser power:1.0 W cm-2, irradiation time: $25 \mathrm{~min}$. Scale bars, $10 \mu \mathrm{m}$. $\mathrm{f}-\mathrm{i}$, The corresponding mean fluorescence intensity collected from SA (f), EC (g), PA (h) and ML (i) under different treatments. All imaging experiments were repeated three times with similar results. Region of interest (ROI) was employed for quantitative assessments of fluorescence intensity, which was calculated by the commercial image analysis software (Leica Application Suite Advanced Fluorescence Lite, LAS AF Lite) and the software of ImageJ. Statistical analysis was performed using a one-way ANOVA analysis. Error bars represent the standard deviation obtained from three independent measurements ( $* \star$ means $p<0.01$, $* \star \star$ means $p<$ $0.001, \star \star \star \star$ means $p<0.0001, n=3$ ). Source data are provided as a Source Data file 

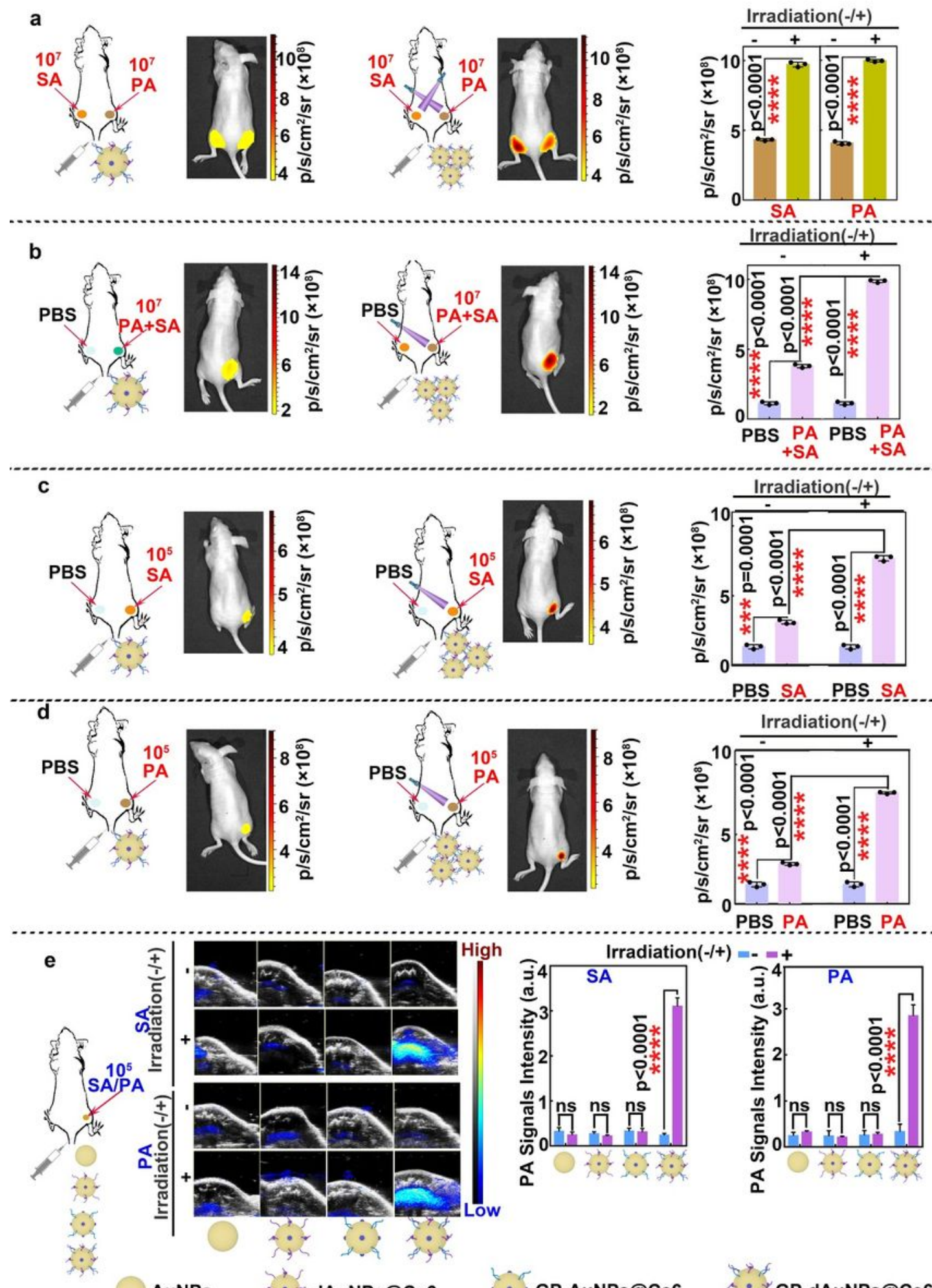

PBS PA

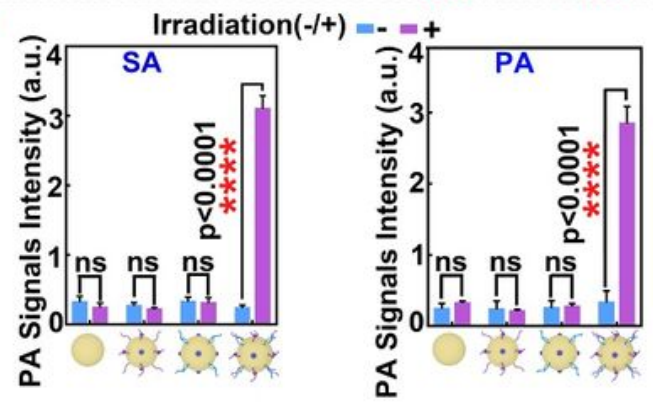

AuNPs

dAuNPs@Ce6

GP-AuNPs@Ce6

GP-dAuNPs@Ce6

Figure 4

Aggregation-enhanced imaging of bacteria in superficial tissues based on the proposed Pac-Man strategy. a, In vivo fluorescence imaging of PA (right side) and SA (left side)-infected sites of mice treated by GP-dAuNPs@Ce6 with or without the irradiation of 405-nm laser and corresponding histograms of fluorescence intensity at two different sites. The bacterial cell concentration during imaging is $\sim 1.0 \times 107$ CFU. b, In vivo fluorescence imaging of the mixture of PA and SA (PA + SA, right side) and PBS (left side)- 
infected sites of mice injected by GP-dAuNPs@Ce6 with or without the irradiation of 405-nm laser and corresponding histograms of fluorescence intensity at two sites. The bacterial cell concentration during imaging is 1.0 $\times 107 \mathrm{CFU}$. c, In vivo fluorescence imaging of SA (right side) and PBS (left side)-treated sites of mice injected by GP-dAuNPs@Ce6 with or without the irradiation of 405-nm laser and corresponding histograms of fluorescence intensity at two sites. The bacterial cell concentration during imaging is $\sim 1.0 \times 105$ CFU. $d$, In vivo fluorescence imaging of PA (right side) and PBS (left side)-infected sites of mice injected by GP-dAuNPs@Ce6 with or without the irradiation of 405-nm laser and corresponding histograms of fluorescence intensity at two sites. The bacterial cell concentration during imaging is $1.0 \times 105 \mathrm{CFU}$. e, In vivo photoacoustic (PA) imaging of SA or PA- infected sites of mice injected by AuNPs, dAuNPs@Ce6, GP-AuNPs@Ce6 or GP-dAuNPs@Ce6 with or without the irradiation of 405-nm laser and corresponding histograms of PA signal intensity at two sites. The bacterial cell concentration during PA imaging is $\sim 1.0 \times 105 \mathrm{CFU}$. All imaging experiments were repeated three times with similar results. Statistical analysis was performed using a one-way ANOVA analysis. Error bars represent the standard deviation obtained from three independent measurements ( ${ }^{\star * \star}$ means $p<0.001$, $\star \star \star \star$ means $p<0.0001$, ns means no significance, $n=3$ ). The cartoons are created by Dr. Houyu Wang. Source data are provided as a Source Data file 
a
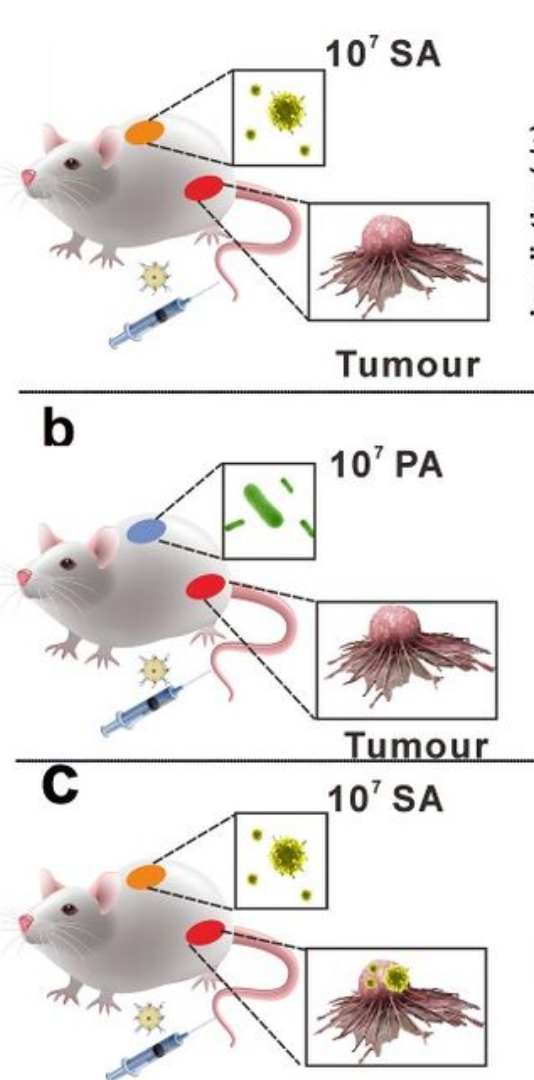

Tumour $+10^{7}$ SA
Fluorescence imaging
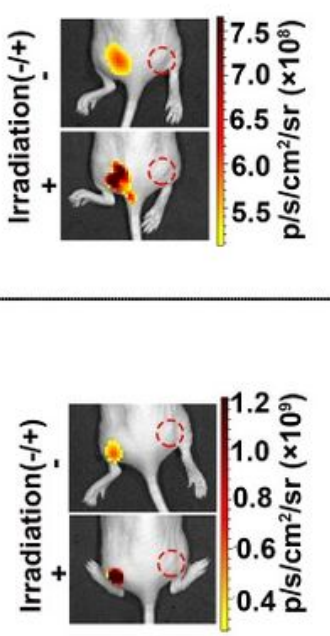

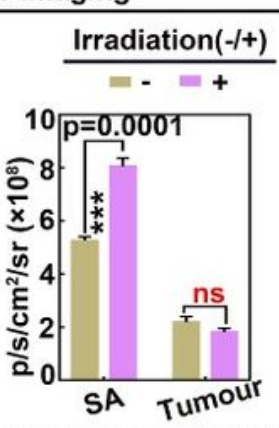

Photoacoustic imaging
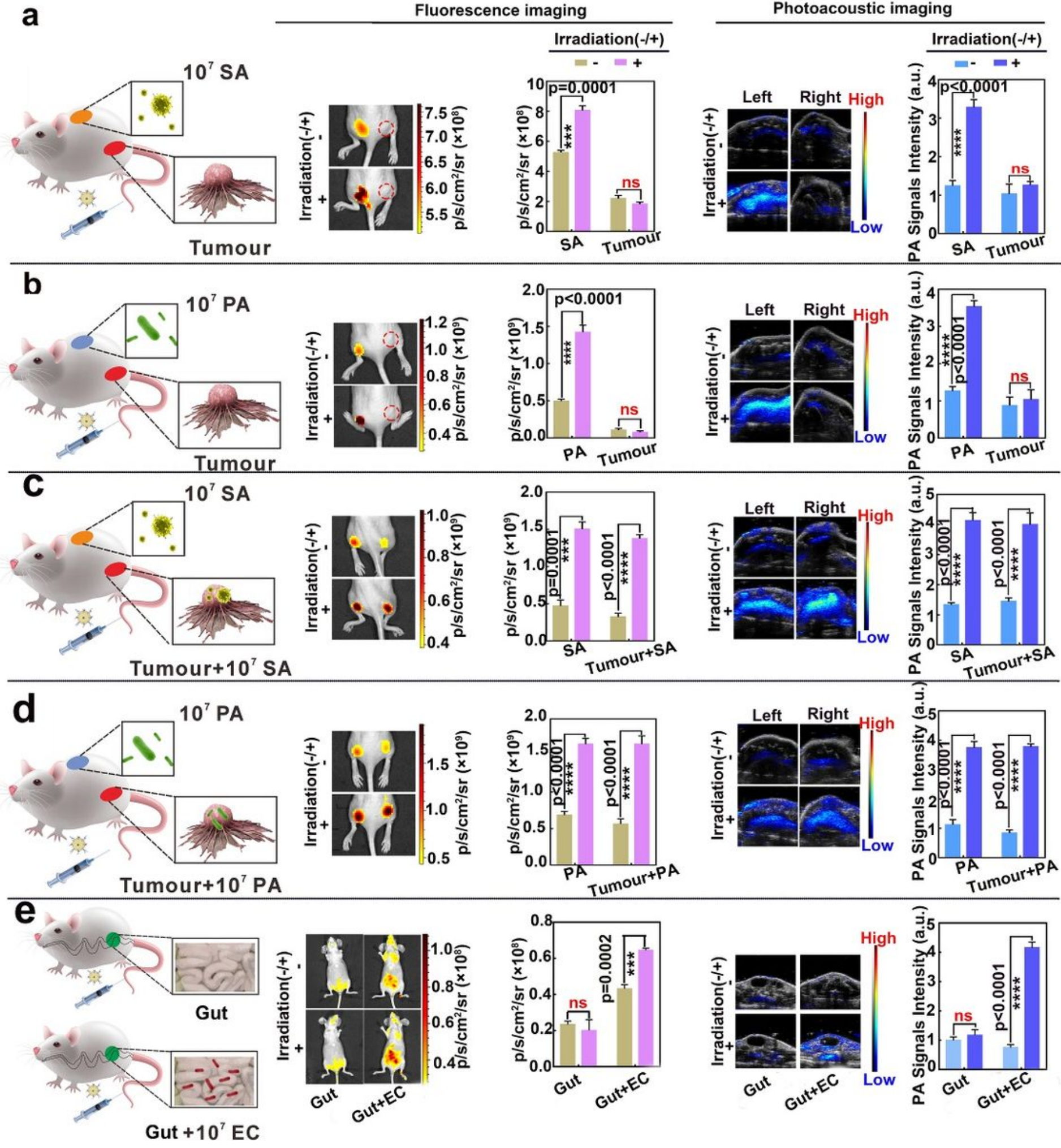

Figure 5

Aggregation-enhanced imaging of bacteria in tumour and gut based on the developed Pac-Man strategy. a, In vivo imaging of SA-infected sites and tumour sites (containing no SA) of mice treated by GPdAuNPs@Ce6 with or without the 405-nm laser irradiations and corresponding histograms of fluorescence or photoacoustic intensity at two different sites. b, In vivo imaging of PA-infected sites and tumour sites (containing no PA) of mice treated by GP-dAuNPs@Ce6 with or without the irradiation of 
405-nm laser irradiations and corresponding histograms of fluorescence or photoacoustic intensity at two different sites. c, In vivo imaging of SA-infected sites and tumour sites (containing SA) of mice treated by GP-dAuNPs@Ce6 with or without the irradiation of 405-nm laser irradiations and corresponding histograms of fluorescence or photoacoustic intensity at two different sites. $d$, In vivo imaging of PA-infected sites and tumour sites (containing PA) of mice treated by GP-dAuNPs@Ce6 with or without the irradiation of 405-nm laser irradiations and corresponding histograms of fluorescence or photoacoustic intensity at two different sites. e, In vivo imaging of gut and EC-injected gut of mice treated by GP-dAuNPs@Ce6 with or without the irradiation of 405-nm laser irradiations and corresponding histograms of fluorescence or photoacoustic intensity at two different sites. The bacterial cell concentration during imaging is $\sim 1.0 \times 107 \mathrm{CFU}$. All imaging experiments were repeated three times with similar results. Statistical analysis was performed using a one-way ANOVA analysis. Error bars represent the standard deviation obtained from three independent measurements ( ${ }^{\star \star \star}$ means $p<0.001$, $* \star \star \star$ means $p<0.0001$, ns means no significance, $n=3$ ). The cartoons are created by Dr. Houyu Wang. Source data are provided as a Source Data file 

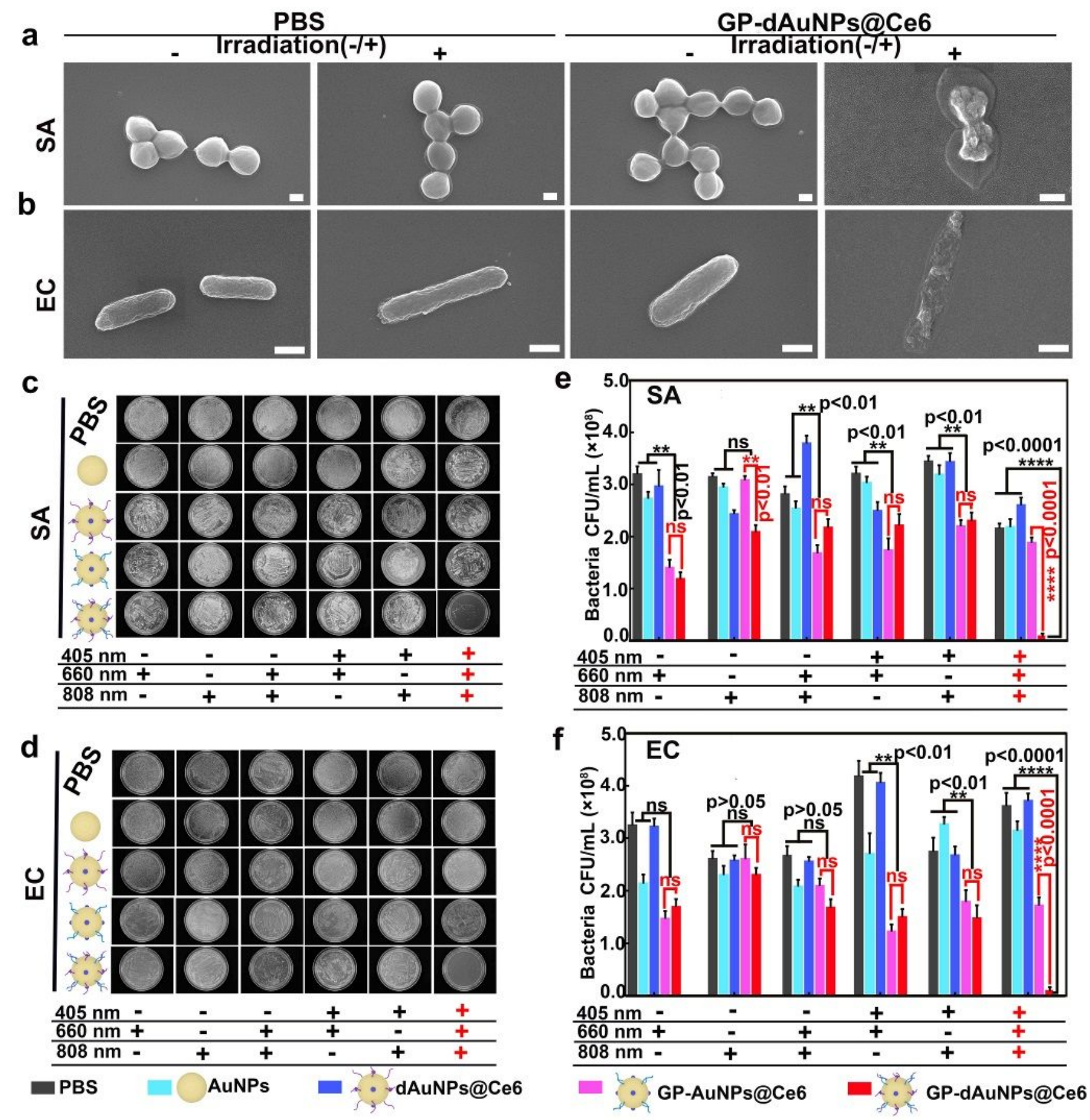

Figure 6

Aggregation-enhanced in vitro antibacterial activity based on Pac-Man strategy. a-b, SEM images of SA (a) or EC (b) treated with PBS or GP-dAuNPs@Ce6 with or without irradiation. Scale bars, $300 \mathrm{~nm}$. c, Photographs of agar plates of SA treated by PBS, AuNPs, dAuNPs@Ce6, GP-AuNPs@Ce6 and GPdAuNPs@Ce6 with different irradiations of 405, 660 and 808 nm laser. e, Corresponding histograms of bacterial amounts of SA bacteria treated by PBS, AuNPs, dAuNPs@Ce6, GP-AuNPs@Ce6 and GPdAuNPs@Ce6 with different irradiation of the 405, 660 and 808 nm laser. d, Photographs of agar plates of EC treated by PBS, AuNPs, dAuNPs@Ce6, GP-AuNPs@Ce6 and GP-dAuNPs@Ce6 with different 
irradiation of 405, 660 and $808 \mathrm{~nm}$ laser. f, Corresponding histograms of bacterial amounts of EC treated by PBS, AuNPs, dAuNPs@Ce6, GP-AuNPs@Ce6 and GP-dAuNPs@Ce6 with different irradiations of the 405, 660 and $808 \mathrm{~nm}$ laser. 405-nm laser:1.0 W cm-2, 25 min; 660-nm laser:12 mW cm-2, 5 min; 808-nm laser:1.0 W cm-2, $5 \mathrm{~min}$. All imaging experiments were repeated three times with similar results. Statistical analysis was performed using a one-way ANOVA analysis. Error bars represent the standard deviation obtained from three independent measurements ( $* *$ means $p<0.01$, $\star \star \star$ means $p<0.001$, $\star \star \star \star *$ means $p<0.0001$, ns means no significance, $n=3$ ). Source data are provided as a Source Data file

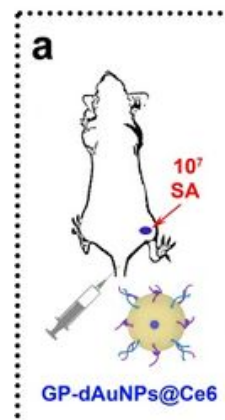

b

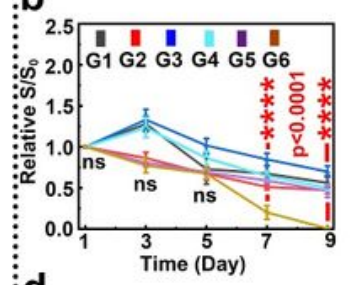

dd

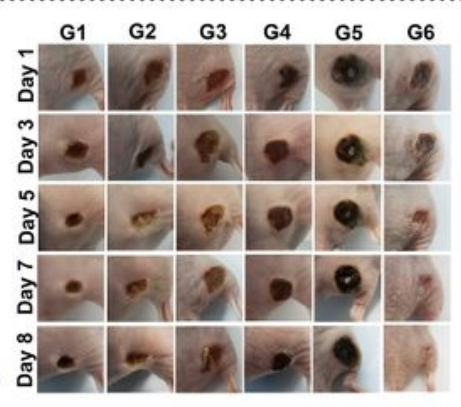

C
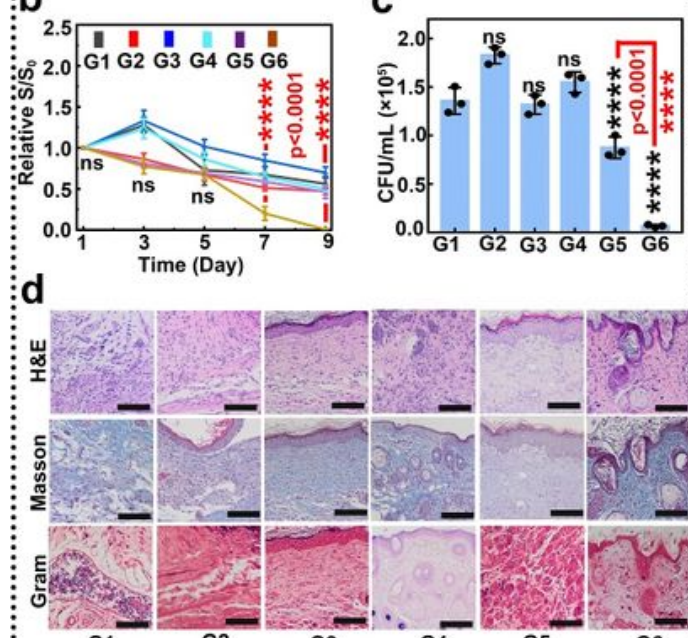
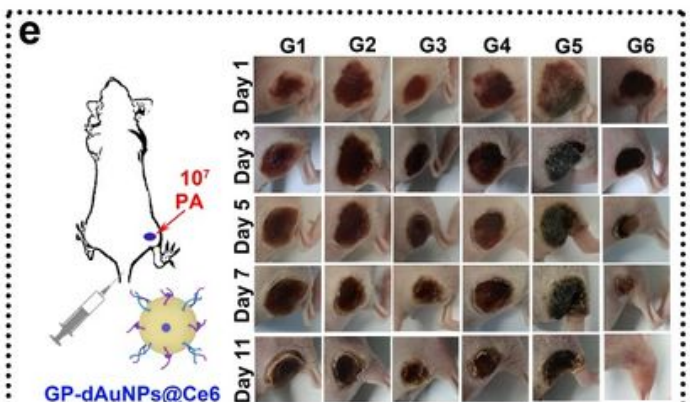

$\mathbf{f}$
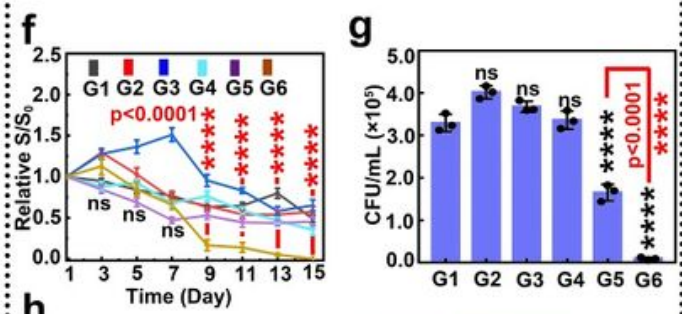

$\mathbf{h}$

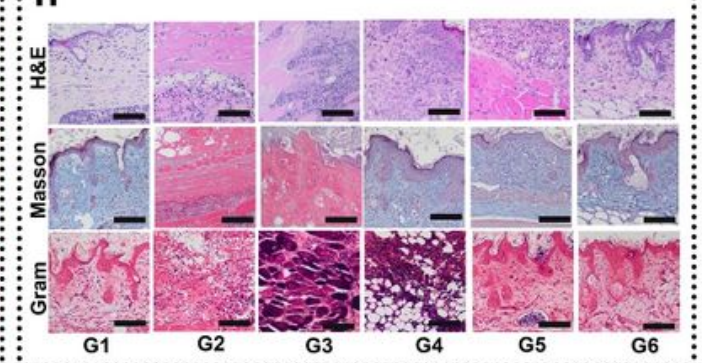

\begin{tabular}{|c|c|c|c|c|c|c|}
\hline & G1 & G2 & G3 & G4 & G5 & G6 \\
\hline $405 \mathrm{~nm}$ & - & - & - & + & + & + \\
\hline $660 \mathrm{~nm}$ & + & - & + & + & - & + \\
\hline $808 \mathrm{~nm}$ & - & + & + & - & + & + \\
\hline
\end{tabular}

i

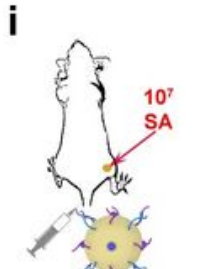

GP-dAuNPs@Ce6

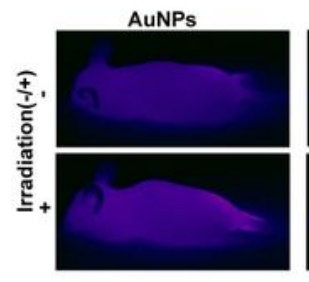

dAuNPs@Ce6

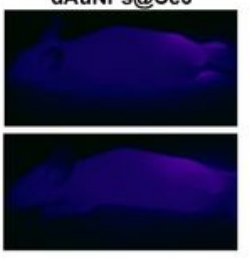

AuNPs
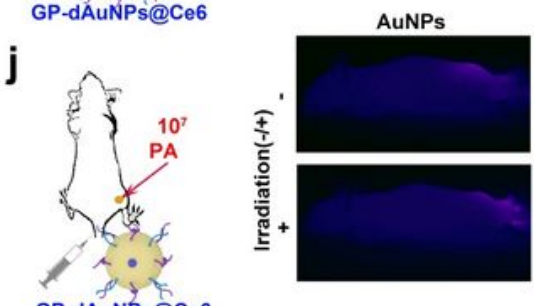

dAuNPs@Ce6

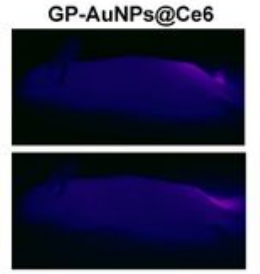

GP-AuNPs@Ce6
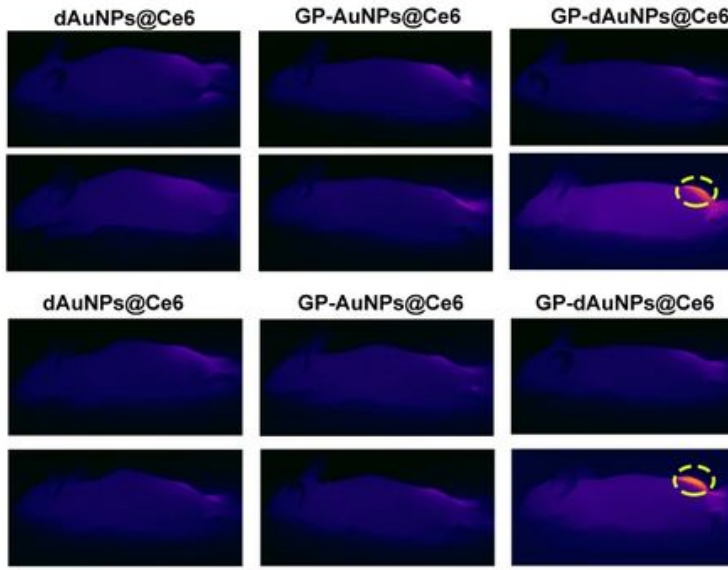

GP-dAuNPs@Ce6

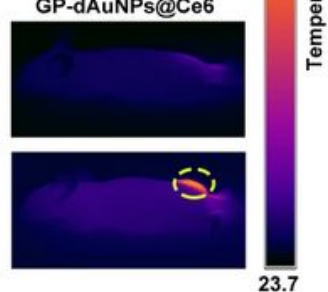




\section{Figure 7}

Aggregation-enhanced in vivo antibacterial activity based on Pac-Man strategy. a, Representative photographs of SA-infected mice injected with GP-dAuNPs@Ce6 with different irradiations of 405, 660 and $808 \mathrm{~nm}$ laser. b, Corresponding time-dependent relative wound area (S/S0) SA-infected mice. c, Bacterial counts (CFU mL-1) excised from the SA-infected tissues of mice at 8-day post-injection. $d$. Corresponding histological images of SA-infected skin tissues of mice at 8-day post-injection. Scale bars, $50 \mu \mathrm{m}$. e, Representative photographs of PA-infected mice injected with GP-dAuNPs@Ce6 with different treatments of 405, 660 and $808 \mathrm{~nm}$ laser irradiation. $\mathrm{f}$, Corresponding time-dependent relative wound area (S/S0) PA-infected mice after different treatments. g, Bacterial counts (CFU mL-1) excised from the PAinfected tissues of mice at 11-day post-injection. h. Corresponding histological images of PA-infected skin tissues of mice at the 11-day post-injection. Scale bars, $50 \mu \mathrm{m}$. The mice in group 1 (G1) are treated with GP-dAuNPs@Ce6+660-nm laser (12 mW cm-2, 5 min); The mice in group 2 (G2) are treated with GPdAuNPs@Ce6+808-nm laser (1.0 W cm-2, 5 min); the mice in group 3 (G3) are treated by GPdAuNPs@Ce6+660-nm laser (12 mW cm-2, $5 \mathrm{~min})+808-\mathrm{nm}$ laser (1.0 W cm-2, $5 \mathrm{~min}$ ); the mice in group 4 (G4) are treated by GP-dAuNPs@Ce6+405-nm laser (1.0 W cm-2, 25 min)+660-nm laser (12 mW cm-2, 5 min); the mice in group 5 (G5) are treated by GPdAuNPs@Ce6+405-nm laser (1.0 W cm-2, 25 min)+ 808$\mathrm{nm}$ laser (1.0 W cm-2, $5 \mathrm{~min})$; the mice in group 6 (G6) are treated by the GP-dAuNPs@Ce6+405-nm laser (1.0 W cm-2, $25 \mathrm{~min})+660-\mathrm{nm}$ laser (12 mW cm-2, $5 \mathrm{~min})+808-\mathrm{nm}$ laser (1.0 W cm-2, $5 \mathrm{~min}$ ). i-j, In vivo photothermal imaging of SA (i) or PA (j)-infected sites on the right back treated by AuNPs, dAuNPs@Ce6, GP-AuNPs@Ce6, or GP-dAuNPs@Ce6. The bacterial cell concentration is 1.0 ×107 CFU during treatment. Statistical analysis was performed using a one-way ANOVA analysis. Error bars represent the standard

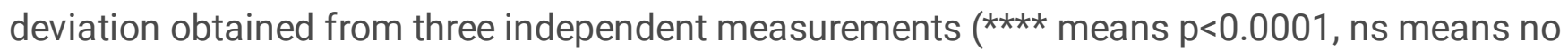
significance, $n=3$ ). Source data are provided as a Source Data file

\section{Supplementary Files}

This is a list of supplementary files associated with this preprint. Click to download.

- FigureS1.xlsx

- Figure2.xlsx

- Figure3.xlsx

- Figure4.xlsx

- Figure5.xlsx

- Figure6.xlsx

- Figure7.xlsx

- FigureS8.xlsx

- FigureS9.xlsx

- nrreportingsummarya2.pdf 
- Nat.Commun.PacManstrategyforimagingandtherapyofbacteriaSPHe.docx 